\title{
«DIVERSE LINGUE, ORRIBILI FAVELLE, MUSICA TRISTE SENZA NOTE»: INTERTESTUALITÀ DANTESCA NEL RAP ITALIANO
}

\author{
Noi che eravamo la stessa persona, \\ Principi senza corona; il tuo ricordo mi abbandona, \\ Il tuo profumo si fa spilli, buca ed ossessiona. \\ Amor, ch'a nullo amato amar perdona, \\ E ti amo come allora
}

\section{DANTE "RAP"}

Come ricordato da Alberto Casadei nel suo recente Dante. Storia avventurosa della «Divina Commedia» dalla selva oscura alla realtà aumentata, ${ }^{1}$ uno dei caratteri piú significativi della potenzialità del poema dantesco, anche in epoca contemporanea, è «la perfetta adattabilità» (2397) alle diverse modalità del narrare, da quelle piú tradizionali (il romanzo e il racconto) a quelle piú moderne e "pop" (fumetto, fiction, serie televisive, ecc.). Seguendo l'argomentazione dello studioso forlivese non si può non riconoscere come la piú autentica dimensione dell'opera sia quindi quella «squisitamente narrativa» (2432), e che - per estensione - l'incisività delle vicende descritte nelle cantiche venga avvertita anche al di là dei suoi specifici aspetti allegorici o delle complessità esegetiche che la contraddistinguono (2438). In altri termini, la Commedia ha descritto un universo che può essere ripensato incessantemente, secondo un processo di riattivazione continuo della materia che si arricchisce, di volta in volta, delle differenti sensibilità culturali che lo reinterpretano (2424): quello che abbiamo di fronte è, pertanto, un testo che oggi, nel fitto scenario offerto

\footnotetext{
${ }^{1}$ Casadei 2020. Si cita dall'edizione elettronica, indicando di volta in volta, tra parentesi tonde, la posizione. 
dalla cultura di massa, ha consolidato la propria dimensione iconica e si presta piú che mai a una molteplicità di riusi. ${ }^{2}$

Settecento anni dopo, il testo di Dante continua cosí a costituire, lungo le diverse vie della cultura, un eccezionale «patrimonio di parole, di immagini e di personaggi e di storie condivise»: ${ }^{3}$ non deve quindi stupire che questo specifico carattere si sia manifestato, e continui a manifestarsi, anche nell'italiano cantato. ${ }^{4}$ Una simile circostanza non è certo sconosciuta agli studiosi: numerosi sono ormai, infatti, i contributi sulla presenza della Commedia nelle multiformi manifestazioni della canzone nostrana. Ci limitiamo a una rapida e non esaustiva rassegna dei lavori recenti, aprendo con l'ampio studio di Fabrizio Galvagni sui riflessi dell'opera nel rock (Dante e l'armonia delle sfere. La Commedia, il rock progressivo e altri percorsi); ${ }^{5}$ per il solo biennio 2020-2021 segnaliamo almeno la monografia di Davide Guerra dal titolo «Paolo e Francesca quelli io me li ricordo bene». Echi danteschi nella canzone italiana ${ }^{6}$ il saggio di Francesca Bravi dedicato ai riflessi del poema nelle canzoni di Vinicio Capossela (La "Marina Commedia" di Vinicio

2 Sulla presenza di Dante e della sua opera nelle diverse forme della cultura di età contemporanea si vedano soprattutto Scorrano 1976, Baranski 1986: 343-76, Scorrano 1994, Casadio 1996, Pegorari 2000, De Rooy 2003, Cotugno 2009, Pegorari 2010: $115-$ 47, Pegorari 2012, Casadei 2013: 145-80, De Martino 2013, Grignani 2014: 69-100, Cotugno-Gargano 2016, Antonelli-Milone 2021: 318-35, Conti 2021, Coveri 2021b: 12-5, Pertile 2021. La rassegna di studi piú completa sulla fortuna internazionale di Dante nei diversi codici culturali secoli XIX-XXI è contenuta nella sezione "Dante contemporaneo" della rivista «Dante. Rivista di studi su Dante Alighieri», curata, dal 2007, da Daniele Maria Pegorari; a questa si rimanda per ulteriori e dettagliate informazioni. Molto utili sono inoltre i numerosi saggi contenuti nei diversi fascicoli di Parole Rubate. Rivista internazionale di studi sulla citazione (in open access al link http://www.parolerubate.unipr.it/). Si vedano infine i fondamentali contributi contenuti in «Dante e l'arte», progetto dell'Institut d'Estudis Medievals (Universitat Autònoma de Barcelona): i volumi a oggi pubblicati sono dedicati a "Dante e il teatro" (2014), "Il sinfonismo" (2015), "Dante e il cinema" (2016), "Dante e la danza" (2017), "Dante e il fumetto" (2018), "Dante e il rock" (2019).

${ }^{3}$ Beccaria 2021: 148-9.

${ }^{4}$ Sulla lingua della canzone in generale si rimanda almeno a Coveri 1992, Accademia degli Scrausi 1996, Coveri 1996, Antonelli 2005, Cartago 2008, Antonelli 2010, Coveri 2011, Coveri-Diadori 2020.

${ }^{5}$ Galvagni 2012.

${ }^{6}$ Guerra 2020. 
Capossela. Canzone d'autore e letteratura di viaggi in Marinai, profeti e balene), ${ }^{7}$ lo speciale Treccani di Federico Della Corte su alcune schegge infernali nella tradizione cantautorale (Can₹oni dell'Inferno), ${ }^{8}$ il volumetto Dante pop e rock di Trifone Gargano, ${ }^{9}$ che riprende e amplia il precedente Dante $p \circ p$. Canzoni e cantautori, ${ }^{10}$ e i preziosi contributi di Lorenzo Coveri. ${ }^{11}$ Dello studioso ligure, in particolare, segnaliamo il recente Dante nelle canzoni, ampio e documentato sguardo d'insieme sulla presenza di Dante e della sua opera nel complesso panorama canzonettistico italiano moderno contenuto in Dante, l'italiano, volume a cura di Giovanna Frosini e Giuseppe Polimeni, pubblicato dall'Accademia della Crusca in occasione della XXI Settimana della Lingua Italiana nel Mondo: ${ }^{12}$ lo studio ricostruisce con precisione le coordinate di una propensione - il riecheggiamento, puntuale o variamente rielaborato, di passi della Commedia e i riferimenti al suo autore che, a partire dai primi echi rintracciabili in alcuni canti della tradizione preunitaria e fine ottocentesca, si fa via via piú consistente nel corso del secondo Novecento, grazie in particolare alle diverse correnti della canzone d'autore (tra tutte, quella della cosiddetta "seconda generazione"), per approdare infine ai generi piú popolari del nuovo millennio - in primo luogo tra i giovani fruitori -, il rock e il pop, 1 "“indie" e il rap. ${ }^{13}$

Facendo leva sull'opportunità, oggi largamente condivisa, dello studio di Dante «al di là delle fissità e degli stereotipi», ${ }^{14}$ e senza al contempo trascurare «i rischi del dantismo "di massa"», ${ }^{15}$ le pagine che seguono intendono fornire - giunti ormai quasi al termine delle celebrazioni per i settecento anni dalla morte del poeta - un ulteriore contributo in tal senso

${ }^{7}$ Bravi 2020: 59-72.

${ }^{8}$ Della Corte 2021.

${ }^{9}$ Gargano 2021.

${ }^{10}$ Gargano 2018.

${ }^{11}$ Coveri 2021a, Coveri 2021b e soprattutto Coveri 2021c.

${ }^{12}$ Cf. Frosini-Polimeni 2021a.

${ }^{13}$ Sul citazionismo nella canzone si veda almeno il fondamentale Ciabattoni 2016; cf. inoltre Talanca 2017 e Polese 2019.

${ }^{14}$ Frosini-Polimeni 2021b: 7.

${ }^{15}$ Sui «rischi del dantismo "di massa"» e, in particolare, della «magnificazione del mediocre» si rimanda alle lucidissime riflessioni contenute in Pegorari 2018. 
attraverso una trattazione limitata all'illustrazione dei principali meccanismi che regolano la citazione della Commedia nell'ultima delle correnti appena evocate, il rap.

Senza alcuna ambizione di ricostruzione storico-artistica, ricordiamo qui in estrema sintesi che il principale prodotto musicale della cultura hip hop giunge in Italia dagli Stati Uniti nel corso della seconda metà degli anni Ottanta del Novecento con il fenomeno delle "posse", ${ }^{16}$ ottenendo un primo, grande successo nei primi anni del decennio successivo grazie ad artisti come Jovanotti e Frankie Hi-nrg e a formazioni quali Sangue Misto e Articolo 31:17 dopo una "fase di stanca" collocabile tra la fine del secolo XX e l'alba del nuovo millennio, torna a beneficiare, a partire dal 2010, di un nuovo periodo di popolarità che ne ha trasferito, in anni recenti, il raggio d'azione nel territorio del "mainstream".

Se i primi artisti caratterizzavano i loro testi per la presenza quasi esclusiva di storie di vita quotidiana ambientate nelle periferie degradate dei centri urbani maggiori, ${ }^{18}$ le ultime generazioni si sono parzialmente rinnovate, differenziandosi talvolta dai loro modelli per una maggiore varietà tematica, continuando nondimeno a rivendicare in linea di massima la propria appartenenza, reale o ideale, alla cultura della strada. Non infrequenti sono inoltre, fin dagli esordi, le correnti minoritarie che attraversano il movimento, spesso ottenendo i risultati piú originali sul fronte creativo: in tal senso andrà almeno riconosciuto, in una posizione di particolare rilievo, il cosiddetto rap letterario o "letteraturap", filone recente rappresentato da artisti quali Rancore, Murubutu e Claver Gold, ${ }^{19}$ carat-

${ }^{16}$ Il termine posse (prestito dell'ingl. posse, propr. 'gruppo di persone, spec. armate', a sua volta dal lat. POSSE 'potere') è gergalismo con il quale vengono denominate in Italia le prime formazioni musicali rap e raggamuffin alternative, principalmente attive nei centri sociali delle città e caratterizzate da chiara connotazione politica; tra le componenti principali ricordiamo almeno il collettivo romano Onda Rossa Posse, l'Isola Posse All Star, di matrice bolognese, i salentini Sud Sound System e i napoletani 99 Posse.

${ }^{17}$ Cf. Accademia degli Scrausi 1996: 285-356 e Scholz 2006: 69-91.

${ }^{18}$ Cf. ibi: 285-99.

${ }^{19}$ Di Murubutu (nome d'arte di Alessio Mariani) e Claver Gold (Daycol Orsini), in particolare, occorre ricordare il "concept album" Infernum, pubblicato nel 2020, un ambizioso progetto di riscrittura contemporanea della cantica attraverso una progressione di brani che ripercorre - come si vedrà nel corso del saggio - il viaggio di Dante tra $\mathrm{i}$ dannati. 
terizzato da soluzioni tematiche e stilistiche in grado di coniugare le sonorità dell'hip hop a contenuti che tendono al dominio della letteratura tradizionale. $^{20}$

Il rap è quindi un genere assai parcellizzato, in continua e rapida evoluzione, che tuttavia presenta alcuni capisaldi che potremmo definire strutturali: è solitamente privo di una linea melodica in senso stretto e risente meno, di conseguenza, delle limitazioni imposte dalla lingua italiana al codice canzone. ${ }^{21}$ D'altro canto, si tratta di un prodotto musicale che nelle sue realizzazioni concrete si modella scrupolosamente su una base ritmica rigida, determinata da «successioni regolari di accenti linguistici (indotti dal ritmo sottostante), invece che procedere in base alle sillabe su un ritmo piú libero».2 ${ }^{22}$ "vincolo accentuale" non costituisce tuttavia una limitazione alla libertà compositiva, che è invece prerogativa tipica di altri generi fondati sulla fissità delle rime e sul verso breve: al contrario, il brano rap, proprio in quanto costituito da uno sviluppo testuale ampio e articolato, che «per l'orecchio non [va] mai a capo», ${ }^{23}$ si configura come una sorta di poema in prosa, affermandosi quindi nel panorama canzonettistico come campo privilegiato del racconto. In aggiunta andrà ancora osservato che le canzoni dell'hip hop italiano delle diverse generazioni manifestano, come noto, una cura particolare per la resa formale: al di là dell'esibita estrosità lessicale, sul versante stilistico riconosciamo quindi l'impiego costante delle figure di suono e della ripetizione (soprattutto rima, allitterazione, assonanza, paronomasia, anafora), oltre a enumerazioni e accumuli,

${ }^{20}$ Si riporta di seguito il frammento di un'intervista a Murubutu, al contempo professore di Liceo e rapper di successo, reputata in tal senso significativa: «Ho unito sonorità tipiche dell'hip hop alla volontà di veicolare contenuti culturali, il che andava di pari passo con la mia attività di insegnante. Ho iniziato a rivolgermi al pubblico del rap, in maggioranza ragazzi, con contenuti che respingevano a scuola ma che potevano accogliere attraverso la musica» («Alessio Mariani: “Io, il professore che insegna a tempo di rap"», Repubblica, 28 novembre 2016, consultabile al link https:/ /www.repubblica.it/cultura/2016/11/28/news/alessio_mariani_io_il_professore_che_insegna_a_tempo_di_ra P_-153006620/); numerosi spunti interessanti a riguardo sono reperibili inoltre nelle dichiarazioni rilasciate nel 2018 dallo stesso rapper al network indipendente "La scimmia pensa" (https://www.lascimmiapensa.com/2018/06/29/intervista-murubutu/).

${ }^{21}$ Cf. almeno Accademia degli Scrausi 1996: 314.

22 Zuliani 2018: 118.

${ }^{23}$ Accademia degli Scrausi 1996: 315. 
giochi del significante, scioglilingua, riformulazioni e manipolazioni, per lo piú a partire da frasi fatte ed espressioni idiomatiche o proprie della nostra memoria collettiva. ${ }^{24}$

Non andrà infine trascurato un ultimo aspetto: raccogliendo in parte l'eredità del rock, il rap ha avuto, e ha tuttora, consistenti effetti culturali, sociali e linguistici sui costumi di molti giovani, ${ }^{25}$ ancora di piú sui giovani di oggi, che possono beneficiare degli indubbi vantaggi offerti dalle diverse modalità di fruizione digitale della canzone, e soprattutto dei suoi messaggi e delle sue parole. Negli ultimi anni si assiste infatti, nel panorama internazionale, a una evidente operazione di riconquista della dimensione testuale dei brani grazie alla creazione di alcune piattaforme - si pensi in particolare a Genius (in precedenza Rap Genius) ${ }^{26}$ - che contribuiscono, con esiti prima sconosciuti, alla diffusione dei testi del rap, alla loro comprensione e alla loro interpretazione, trasformando - mediante un'esperienza di ascolto, combinata alla lettura, piú coinvolgente e profonda - le abitudini musicali di milioni di individui.

Alla luce di queste considerazioni preliminari, il sistema musicale dell'hip hop si preannuncia come uno spazio di osservazione di sicuro interesse per la valutazione della ricezione del messaggio dantesco nell'ambito della moderna canzone italiana. Dal punto di vista metodologico, l'indagine sul "Dante rap" che viene illustrata nelle pagine che seguono è stata realizzata

\footnotetext{
${ }^{24}$ Cf. ibi: 308-13.

${ }^{25}$ Sul rapporto tra formazione culturale giovanile e musica si rimanda soprattutto a Madrussan 2021.

${ }^{26}$ La piattaforma Genius (https://genius.com/), regolarmente consultata nella realizzazione di questo studio, si fonda su contenuti generati dagli stessi utenti mediante l'impiego di un format simile a quello di Wikipedia. Non limita tuttavia il proprio apporto alla pubblicazione dei testi dei brani, ma genera un luogo virtuale nel quale vengono realizzate operazioni di "annotazione" (glosse, interpretazioni di singole voci, versi o intere canzoni) oltre a costanti scambi di opinioni tra gli ascoltatori di tutto il mondo (o, nelle versioni delle singole nazioni, di uno specifico paese): si presenta quindi come una grande comunità di giovani che divulgano testi, li discutono, li commentano, riportando in definitiva le parole al centro dell'attenzione. Si tratta di un fenomeno in forte sviluppo, del quale sono a conoscenza gli stessi artisti, a loro volta spesso coinvolti in prima persona nelle attività del sito.
} 
attraverso l'analisi di un corpus testuale caratterizzato da massima ampiezza secondo la prospettiva cronologica (l'arco temporale definisce un periodo di circa trent'anni, che va dall'inizio degli anni Novanta al 2021), successivamente sottoposto a selezione sulla base del criterio della popolarità: sono stati cioè presi in esame i soli brani entrati nelle classifiche ufficiali della piattaforma di streaming musicale piú popolare, Spotify, riconducibili ad artisti con un numero di ascolti mensili minimo (centomila); in sede di commento, un'attenzione specifica è stata poi rivolta alla produzione degli ultimi due decenni, la piú sensibile - come si noterà - al recupero della lezione offerta dalla Commedia.

\section{RIFLESSI ONOMASTICI}

Come ricordato da Luca Serianni, «la forza d'urto dei lacerti ${ }^{27}$ danteschi è condizionata, tanto nella lingua letteraria quanto in quella comune, da una serie di fattori: tra questi, i piú determinanti paiono essere la popolarità di alcuni canti, l'«aggancio» a particolari episodi, la «trasparenza» di voci, espressioni, modi di dire e la celebrità di alcuni personaggi. In questa prima sezione l'attenzione ricadrà sull'individuazione e sull'analisi dei riflessi onomastici generati, nei brani sottoposti a spoglio, da alcune delle piú autorevoli figure della Commedia, a partire, naturalmente, da quella del suo autore. ${ }^{28}$

\subsection{Dante}

Il nome di Dante è presenza salda nel rap; si può affermare, senza correre il rischio di scadere in mere generalizzazioni, che quasi tutti i principali rappresentanti del genere musicale di ieri e di oggi, da Fabri Fibra ${ }^{29}$ a Mar-

\footnotetext{
${ }^{27}$ Serianni 2013: 292.

${ }^{28}$ Le citazioni sono tratte da Commedia (Chiavacci Leonardi); d'ora in poi If o $\mathrm{Pg}$ o $P d$.

${ }^{29}$ «Non faccio musica leggera né pesante, / faccio il rap nauseante / come a scuola per me Dante» (Tu cosí bella non ce l'hai, 2007).
} 
racash, ${ }^{30}$ dalla Dark Polo Gang ${ }^{31}$ a Gue Pequeno, ${ }^{32}$ dai Club Dogo ${ }^{33}$ a Rkomi, ${ }^{34}$ hanno evocato il poeta inserendolo nei loro testi adottando, di volta in volta, soluzioni diverse. Una presenza che viene registrata con regolarità fin dalle origini dell'hip hop italiano (almeno a partire da Kaos One, una delle voci piú popolari degli anni Novanta: «È meglio che mi lasci stare, / ho altro da fare che / sentire te che parli, / sentire il tuo racconto: / sei pesante come Dante, / non ti affronto piú», Fino alla fine, 1996) e che si caratterizza per un incremento apprezzabile nel corso dei decenni successivi. In poco piú di un ventennio, dal 2000 a oggi, sono infatti reperibili circa un centinaio di occorrenze del solo nome proprio dello scrittore fiorentino, ${ }^{35}$ con una concentrazione particolare (una trentina di casi) nel quadriennio 2018-2021; di seguito una serie minima di esempi degli ultimi anni:

In un mare di carte, qua, in viaggio tra un passo del Tasso e un canto di Dante, tra un diario di Kafka e una fiaba di Márquez, e fra una trama di Gadda e un passaggio di un saggio di Sartre, avvolti dall'alba compatta come dentro a una saga di Fante.

(Carlo Corallo, I maestri, pt. 2, 2018)

Nella selva di Dante

con un'erba alienante, che mi prenda all'istante, che mi reggan le gambe.

(Ozymandias, Jeeg Robot, 2019)

${ }^{30}$ «Un messaggio importante: come Dante / giú negli inferi, vedo la commedia dei media» (Quando sarò morto, 2011).

${ }^{31}$ «La tua collana suscita domande, / scateno l'Inferno come Dante» (Che bello essere dark, 2018).

32 «La strada per me è stata come per Dante Beatrice» (Maledetto, 2019).

33 «Da ragazzo ho letto Dante, l'inferno pazzo dietro ogni passo; / ora rappo sulla techno per contrappasso» (D.O.G.O., 2006).

${ }^{34}$ «Non sono Dante, l'Inferno, il mio inferno è un altro» (Paradiso vs. Inferno, 2021).

${ }^{35}$ Sono state prese in considerazione le sole occorrenze connesse con chiarezza al poeta, escludendo gli altri casi (ad es. la presenza dell'antroponimo nei riferimenti ai nomi di vie, piazze, ecc.). 
Sono mille minuti che aspetto qua dentro, non mi chiamo Dante ma questo è l'inferno.

(Myss Keta, Rider bitch, 2020)

Sono Dante a disagio con Bea, quello che se non è stressato non crea.

(Caparezza, Azzera Pace, 2021)

I rapper piú "affezionati” al nome di Dante sono probabilmente Caparezza e Tedua. Per l'artista pugliese l'autore della Commedia è riferimento che potremmo definire stabile, utilizzato negli anni in contesti eterogenei e con differenti finalità; oltre al caso segnalato nella lista precedente, si riproducono qui anche alcune occorrenze anteriori: «Sei la mia musa ispiratrice: / io Dante, tu Beatrice» (Ti clonerò, 2000); «E pensare che per Dante questo era il "bel paese là dove 'l sí sona"» (Goodbye Malinconoia, 2011). Di particolare interesse, nella sua ampia discografia, è il brano Argenti vive, pubblicato nel 2014; al suo interno l'Alighieri viene in piú circostanze nominato da Filippo Argenti, protagonista dell'episodio cantato, tramutandosi in "bersaglio" del dissing, vale a dire dell'attacco tagliente, ${ }^{36}$ tutto rime e giochi di parole, dell'anima dannata: ${ }^{37}$ «Ciao Dante, ti ricordi di me? Sono Filippo Argenti, / il vicino di casa che nella Commedia ponesti tra questi violenti»; «Inutile che decanti l'amante, Dante, provochi solo cali di libido: / il mondo non è dei poeti, il mondo è di noi prepotenti»; «Persino tu che mi anneghi a furia di calci sui denti / ti chiami Dante Alighieri,

${ }^{36}$ Cf. https://www.treccani.it/vocabolario/dissing_res-5a9cc2ef-8997-11e8-a7cb00271042e8d9_\%28Neologismi\%29/.

${ }^{37}$ Il brano fa parte di Museica, un album nel quale ogni componimento viene sviluppato a partire da un'opera pittorica (il nome Museica è, del resto, un neologismo in cui si riconoscono le parole museo e musica). Utili in tal senso sono le considerazioni con le quali lo stesso Caparezza introduce la canzone nel booklet del disco: «Tutti prima o poi ci siamo imbattuti in una illustrazione di Gustave Doré. Quelle nella Divina Commedia, per esempio, sono le sue ma molti lo ignorano, cosí come ignorano che il vigoroso culturista da lui dipinto nel fiume Stige sia Filippo Argenti, vicino di casa di Dante. Il sommo poeta distrugge il dirimpettaio con un "dissing" violentissimo: ora il microfono passa ad Argenti». Cf. anche Guerra 2020: 58-61 e Coveri 2021c: 350-1. Uno studio accurato sui riferimenti culturali all'interno di Museica è in Miglietta 2019. 
ma somigli negli atteggiamenti / a Filippo Argenti»; «Guardati le spalle, caro Dante, è pieno di bruti: / tutti i grandi oratori sono stati fatti fuori / da signori, violenti e nerboruti». ${ }^{38}$

Nei testi del giovane rapper di origini genovesi Tedua, invece, il nome dantesco si combina spesso con quello dell'artista, generando l'ardito composto DanTedua: ${ }^{39}$

Vieni e balla con il poeta, Dan'Tedua.

(Tedua, Elisir, 2019)

DanTedua, l'inferno:

piú cerchi dell'hula hoop.

(Tedua, Party HH, 2020)

DanTedua all'inferno verso il Paradiso.

(Tedua, Inferno, 2020)

Dante diviene via via piú ricorrente nei testi dei suoi due album successivi, Vita vera mixtape: Aspettando la Divina Commedia (2020) e Don't panic (2021), entrambi fondati sul resoconto di un viaggio attraverso un inferno urbano:

Storie, Divina Commedia, tu chiamami Dante.

(Young rame, John Gotti, 2020)

Dante espia tutti i peccati, ma tu fai la spia.

(Tedua, Gironi, 2020)

${ }^{38} \mathrm{Nel}$ corso del brano l'autore della Commedia è denominato anche, per antonomasia (ma pur sempre in una dimensione polemica), sommo ("Cos’è vuoi provocarmi, sommo? / Puoi solo provocarmi sonno»), vate («Eri te che mi deridevi, devi combattere / ma te la dai a gambe levate: ma quale vate?») e poeta («Poeta, tu mostri lo sdegno a Filippo Argenti»), oltre che con il solo cognome (cf. infra).

${ }^{39}$ Cosí si esprime l'artista a riguardo: «È un gioco di parole che mi è uscito a caso mentre scrivevo. C'è da dire che tutti i fan mi chiamavano sempre "poeta" e allora ho voluto giustificare un po' questa cosa nel mio immaginario» (cf. https://www.outpump. $\mathrm{com} /$ tedua-vita-vera-intervista/). 
Rappo, rappo, dietro al retro esco, vesto Prada, leggo Dante, pago le cene con del contante.

(Tedua, Badman, 2021)

Non manca, come da previsioni, una specifica propensione al richiamo onomastico dantesco nei testi di alcuni esponenti del "cantautorap" ${ }^{40}$ (cf. ad es. «Io sono solo un commediante come Dante, / tu sei solo una come tante», Dargen D’Amico, Ma dove vai (Veronica), 2011) e, con un rilievo piú consistente, in quelli dei rapper del filone "didattico" e letterario: segnaliamo tra questi ultimi, oltre al già citato Carlo Corallo, l'occorrenza del nome d'autore ne Il sentiero dei nidi di ragno (2017) di Lowlow: «Non credo a inferno o paradiso, non ti credo Dante, /credo nella scrittura, immortalità dell'arte». Di contro, il poeta è spesso ricordato anche nei prodotti delle correnti maggiormente esposte ai registri colloquiali piú spinti, all'interno di sequenze non prive di toni triviali: «Ma non avremmo la Divina Commedia / se Dante si fosse prima bombato Beatrice» (J-Ax, Il bello d'esser brutti, 2015); «Un uomo in carrozzella si coddava i buchi neri, / Divina Commedia, Dante Alighieri» (Mc Cavallo, Il ritorno del cavallo vivente, 2018); «Adesso ci provo a dirti qualcosa di nuovo, / mi ti fotto dolcemente, predico il dolce stilnovo: / sí, Dante Alighieri, tirate in alto i bicchieri, / che se mi incazzo devono ingrandire i cimiteri» (Real Talk, Il Tre, 2018).

In diverse circostanze il nome del poeta si accompagna al cognome; quest'ultimo è talvolta in rima: «Niente rimedi per chi è nato oggi e non ieri; / Dante Alighieri, dai, chiamate gli artificieri» (Il tre, Io non sono come te, 2021). Modeste, e limitate al solo Argenti vive di Caparezza, sono invece le occorrenze di Alighieri non preceduto dal nome proprio: «Alighieri, vedi, tremi, mi temi come gli eritemi, eri te che mi deridevi»; «Cosí impari a rimare male di me, io non ti maledirei, ti farei male, Alighieri». ${ }^{41}$ tautorale.

${ }^{40}$ Corrente interna al genere che si ispira, nello stile e nei temi, alla tradizione can-

${ }^{41}$ A differenza di quanto accaduto nella canzone d'autore della prima metà degli anni Settanta: cf. almeno Metrò di Enzo Jannacci («Ma tu non m’hai visto / leggere il giornale / come se fosse scritto da Alighieri», 1970), Talkin sul sesso di Francesco Guccini («anche Alighieri col sesso prendo, e la Divina Commedia a dispense vendo: / la carne in fiamme, peccatori e peccatrici, sensazioni paradisiache», 1973) e soprattutto Alighieri di 
Sul fronte stilistico e retorico, l'antroponimo offre il maggior contributo - ed è requisito ampiamente prevedibile - nelle similitudini (tramite il consueto come o attraverso il piú generazionale tipo) e nei paragoni, con riferimento esplicito prevalente al viaggio nell'oltretomba: «Gli amici veri sono pochi e le ragazze tante, / e tante vivono un viaggione come Dante: / tu giochi da amatore e loro giocan con l'amante» (Albert, Alter ego, 2016); «Sto perdendo le staffe nella selva come Dante» (NDG, Corone d'alloro, 2019); ; «In giro di notte Neda tipo Dante, / voi rap hip-hop, io rap ignorante» (Gemitaiz, Gigante, 2016). ${ }^{43}$ In tal senso, il poeta è talora percepito come modello («La sua scrittura è paragonabile a quella di Dante, / la sua eloquenza è affascinante», Garfo, Rime in are, 2018); in altri casi è alla base di un processo di immedesimazione («Bacio bicchieri, io all'Inferno, Dante Alighieri», Caneda, Seven, 2015, con andamento ellittico), in altri ancora diviene quasi un alter ego dell'artista («La mia roba è scritta da Dante», Zuno Mattia, Il mondo assiste, 2016, con un'imprudente associazione al genericismo roba che provoca un forte contrasto).

Come rilevabile in alcuni dei frammenti appena riprodotti, l'antroponimo è collocato con assiduità in posizione di rilievo a fine verso, spesso in rima (o in contesto allitterante), anche con disposizione degli elementi contraddistinti da omofonia parziale in sequenza rapida e incalzante secondo una propensione connaturata al canone metrico del genere: «Non faccio musica leggera né pesante, / faccio il rap nauseante, / come a scuola per me Dante» (Fabri Fibra, Tu cosí bella non ce l'hai, 2007); «Rasty Killah sfonda il beat con la realtà piú pesante, / la mia musica è assordante, sto al-

Roberto Vecchioni (nella sezione finale, recitata: «da Firenze il discorso si espande a tutto il mondo: diventa universale. Ed è qui che l'Alighieri troneggia in un crescendo di malinconia e passione», 1975).

${ }^{42} \mathrm{Si}$ veda anche l'esempio che segue, caratterizzato dalla costruzione del verso attraverso l'impiego delle "parole tandem" (ossia termini accostati in una struttura ellittica che ricorda l'analogia, cf. Cristalli 2019): «Siete belli pieni di cazzate fresche / come versi, Dante, piante, le foreste» (L'elfo, Caronte, 2018).

${ }^{43}$ Anche nella sequenza nome + cognome: «Come te ho gli stessi pensieri, / sono cresciuto rimanendo un po' lo stesso di ieri; / nel bel mezzo del cammino, come Dante Alighieri, / mi sono perso tra mille domande in questi sentieri» (Cane secco, Love kills, 2014). 
l'inferno come Dante, / facce sfrante a caccia del contante» (RapCore, Melodie crudeli, 2011); «Vita troia, il destino è l'amante, / sul polso ho un diamante, / ho piú strofe di Dante, / scappo dalla volante, / il mio spirito guida ubriaco al volante» (Jesto, Emma, 2015). Raro invece è il suo utilizzo nella composizione di giochi di parole: «Se cercavi qualche cosa di piú interessante / lascia perdere Danti, ${ }^{44}$ leggiti Dante» (Two fingerz, Bukowsky, 2015).

È già stato osservato che il poema dantesco si configura, nel corso del Novecento (e con una propulsione perfino piú intensa nel terzo millennio, principalmente nell'esteso perimetro del "pop"), come paradigma in grado di essere impiegato per scandagliare i destini della contemporaneità, tanto quelli individuali quanto quelli collettivi. Nell'ambito specifico del rap, in diverse occasioni il nome del poeta risuona quindi - come del resto si sarà notato dalla lettura di parte dei passi prodotti in precedenza (e come emergerà dal seguito del contributo) - nell'elaborazione di nuovi inferi, attuali e tangibili; andrà a questo proposito ancora segnalato il recente brano di J-Ax, Voglio la mamma (2021), nel quale viene stabilita una correlazione tra le vicende infernali della prima cantica e alcune delle piú drammatiche circostanze determinate dalla pandemia di Coronavirus: «I test rapidi danno false speranze, / come chi gestisce le crisi lombarde; / musica spenta, suonano solo le ambulanze, / poi per un mese ho visto il vero inferno, altro che Dante». ${ }^{45}$

Se la Commedia è un capolavoro «che porta in sé i segni e la sintesi piú alta del tempo in cui è nato», ${ }^{46}$ il "grande fiorentino" ha dal canto suo ac-

${ }^{44}$ Danti è il soprannome del cantante del duo (Daniele Lazzarin).

${ }^{45}$ Si rileva a latere anche la presenza dell'aggettivo dantesco, associato per lo piú a Inferno: «Inferno dantesco con il contrappasso» (Mad Man, Guarda mamma, 2013), «Ma dentro il peggio, fra’, è un Inferno dantesco» (Lazza, Super Santos Freestyle, 2016), «Siamo solo al primo piano dell'Inferno dantesco» (Side baby, Nulla, 2021); sporadicamente è inoltre posto in corrispondenza con un altro termine chiave (rima, demonio e girone): «Scusa la rima dantesca, / ma quando rappo è sempre pesce in bocca, palla in testa» (Marracash \& Guè Pequeno, Cosa mia, 2016); «Denoto decorso demotivato denominato / Da decenni demonio dantesco dentro dischi» (Lord Madness, M.A.D., 2016); «Tequila, limone, / un altro girone / dantesco, manesco, / io non so bailan» (J-Ax, Fiesta, 2020).

${ }^{46}$ Casadei 2020: 1868. 
quisito, nell'epoca della globalizzazione, la dimensione di un'icona «che si adatta a tanti riusi». ${ }^{47}$ Non può mancare quindi, nel nostro corpus, l'impiego del nome del poeta come emblema identitario di una nazione dalla cultura secolare: «Ero fiero di fare l'italiano / per la storia e l'impero dove abitavo: / Giordano Bruno, da Dante a Da Vinci; Colombo partí da Palos. / Ora vedo uno strano Stato» (Rapstar, Una possibilità, 2012); «Italiano come la pizzica, come la pizza, / come Dante [...], come una lingua» (Ensi, Tutto il mondo è quartiere, 2018).

Allo stesso tempo, tuttavia, il poeta può assumere altre fisionomie, talvolta meno qualificate: c'è infatti il Dante "avversario", cantato da Filippo Argenti (cf. Argenti vive), e quello "noioso", legato all'esperienza scolastica («Non faccio musica leggera né pesante, / faccio il rap nauseante / come a scuola per me Dante», Fabri Fibra, Tu cosí bella non ce l'bai, 2007; «Da ragazzo ho letto Dante, l'Inferno pazzo dietro ogni passo; / ora rappo sulla techno per contrappasso», Club Dogo, D.O.G.O., 2006); in altre circostanze, il poeta viene rievocato per marcare la sua distanza rispetto all'artista rap («Non sono Dante, l’inferno, il mio inferno è un altro» (Rkomi, Paradiso vs. Inferno, 2021), per denigrare la dimensione intellettuale dell'individuo in favore di una prospettiva mondana di ostentazione della ricchezza («Lo sapevo che ce l'avrei fatta, / mi serviva solo diventare grande. / Ora con la musica ci faccio il grano, / e non mi serve di prendere Dante» (Basement café, Basement café next gen, 2019) o, ancora, per piú generiche finalità ironico-scherzose (il pensiero va almeno al Dante «a disagio con Bea» di Caparezza, cf. supra), peraltro già sfruttate in ambito letterario. $^{48}$

\subsection{Beatrice e Virgilio}

Tra gli altri nomi, ${ }^{49}$ minime considerazioni possono essere espresse a proposito di Beatrice e Virgilio. I due personaggi rivelano inevitabilmente un

${ }^{47}$ Ibi: 2392; cf. anche Conti 2021.

${ }^{48}$ Cf. almeno la Beatrice raffigurata da Stefano Benni ne Le Beatrici, 2011 (per una lettura linguistico-stilistica del testo si rimanda a Bellone 2017: 101-9).

${ }^{49}$ Non vengono qui presi in considerazione i nomi dei personaggi, anche principali, della Commedia collocati in specifici canti; per questi cf. infra. 
tasso di popolarità inferiore rispetto all'autore della Commedia: sul fronte onomastico sono infatti poco piú di una decina per entrambi le occorrenze $^{50}$ reperite nei testi scandagliati, quasi tutte collocabili nella produzione rap dell'ultimo decennio.

Beatrice è, nella maggior parte delle occasioni, in sede di rima: «Ma poi chiama lei, forse stasera ci vediamo, dice, / e io passo l'inferno, Beatrice» (Gemitaiz \& Mad Man, Drama, 2014); «Dante violenta Beatrice, / quello che si fa e non si dice» (Ketama \& Sean, Numeri, 2014). Quasi mai collocata in una dimensione storica coerente con la materia dantesca $(« \mathrm{E}$ lei era tutto il sentire, il mio limes sottile, / la mia Beatrice incorporea verso la nuova aurora a venire», Chiodo, Paradiso perduto, 2016), appare per lo piú come facile topos (si veda ad es. il brano Ti clonerò, di Caparezza, supra), anche all'interno di raffronti inattesi («La strada per me è stata come per Dante Beatrice, / i soldi non mi faranno felice», Guè Pequeno, Maledetto, 2019) o in frangenti di stretta attualità che determinano un forte contrasto espressivo («Ma cerco solo Beatrice, no bitch arriviste attorno», Tedua, Paris, 2021).

Il nome della donna ricorre però nel titolo del brano che offre il suo ritratto piú efficace e intenso (Murubutu e Claver Gold, Beatrice, 2020): ${ }^{51}$ nella traccia, l'unica nel panorama canzonettistico italiano a lei interamente dedicata, Dante (Murubutu), sul modello di Vita Nuova II, ${ }^{52}$ riferisce del primo incontro con l'amata, qui anche diretta interlocutrice; da segnalare, nell'attacco - caratterizzato da chiaro incedere lirico -, la precisione offerta dal dato temporale del primo verso («Questo febbraio ti vibra negli occhi verdi») e dalla successiva coordinata spaziale contenuta nel riferimento alla chiesa di Santa Margherita dei Cerchi:

Questo febbraio ti vibra negli occhi verdi,

Già da lontano stupivi per le movenze lente,

\footnotetext{
${ }^{50}$ Alcune di queste in brani nei quali viene ricordato anche il nome del poeta fiorentino.

${ }^{51}$ Il nome è solo nel titolo della traccia; si segnala tuttavia, a testo, l'interessante ipocoristico Bice («Dove il mio cielo è radice muovo ogni passo infelice / tra qualche bella attrice, ma amavo solo Bice»), evidente ripresa di una coppia di versi di E cantava le canzoni, brano del 1978 di Rino Gaetano ( $« \mathrm{E}$ partiva il produttore / con il copione scritto in fretta, / cercava qualche bella attrice, / ma lui amava solo Bice»).
} 
Passavi piano compita fra i vari affreschi

Di Santa Margherita dei Cerchi a Firenze.

In accordo con il "libello", il seguito della prima strofa allude alla trasfigurazione della giovane secondo quel processo di elevazione e di oggettivazione dell'esperienza amorosa che, dalle "nove rime", giungerà a compimento nei canti del Paradiso; in un disegno metrico che procede serrato per l'andamento anaforico ed allitterante di costrutti fondati sulla seconda persona singolare dell'imperfetto eri, l'adesione alla Vita nuova trova conferma almeno nel «segno carminio»e, ancora piú esplicitamente, nel «drappo sanguigno» (cf. VN II: «Apparve vestita di nobilissimo colore, umile e onesto, sanguigno» e soprattutto VN III: «Involta mi parea in uno drappo sanguigno leggeramente»):

Ed eri piú che un sorriso, eri un segno divino,

Eri un segno carminio dentro il cuor;

Ed eri un sogno in cammino

Fra senso e destino ad un tratto su un drappo sanguigno:

Lume al buio nel vuoto, cuore a nudo nel fuoco. ${ }^{53}$

La seconda strofa, interpretata da Claver Gold, trasferisce invece la vicenda dei due protagonisti all'Inferno attraverso un'efficace giuntura rappresentata dal primo verso della sezione - tra Vita nuova e Commedia: «Io ti cercavo negli occhi di chi ti osserva e non dice: ${ }^{54} /$ sotto la terra sinistra qui dista la superficie, / dove il mio cielo è radice muovo ogni passo infelice». Nei versi successivi, Dante richiama l'episodio delle tre fiere di If I, 31-60: merita attenzione in particolare la narrazione dell'incontro con la lince («Dove una lince è lussuria e ferma i miei piani, / io tremo ancora

${ }^{52}$ Citazioni tratte da Vita Nuova (Pirovano-Grimaldi); d'ora in poi VN.

${ }^{53} \mathrm{Il}$ «cuore a nudo nel fuoco» è forse da confrontare con la "maravigliosa visione" descritta in VN III: «E quando elli [Amore] era stato alquanto, pareami che disvegliasse questa [Beatrice] che dormia; e tanto si sforzava per suo ingegno, che le facea mangiare questa cosa [il cuore di Dante] che in mano li ardea».

${ }^{54} \mathrm{E}$ in effetti una probabile, ulteriore eco di VN; cf. infatti il sonetto Tanto gentile e tanto onesta pare: «Tanto gentile e tanto onesta pare / la donna mia quand'ella altrui saluta, / ch'ogne lingua deven tremando muta, / e li occhi no l'ardiscon di guardare» (vv. 1-4). 
al ricordo delle tue mani»), nella quale le parole del poeta parrebbero alludere a un risvolto non esclusivamente platonico della vicenda amorosa. Questa seconda parte si conclude con un rapido rimando a Virgilio, una coppia di versi («Ma tu mandasti un compagno a farmi luce nel viaggio, /a darmi forza e coraggio») che condensa il contenuto di If $\mathrm{I}, 61$ ss.

Il ritornello in terza persona tratteggia infine la modalità della celebrazione della donna, in una costante corrispondenza di strutture sintattiche basate sulla tripartizione dei costituenti (per lo piú bisillabi: al di là di «vita divina», cf. infatti «ogni rima», «ogni riga», «ogni climax», «guida», «ogni sfida», ecc.) introdotte nuovamente dall'anafora:

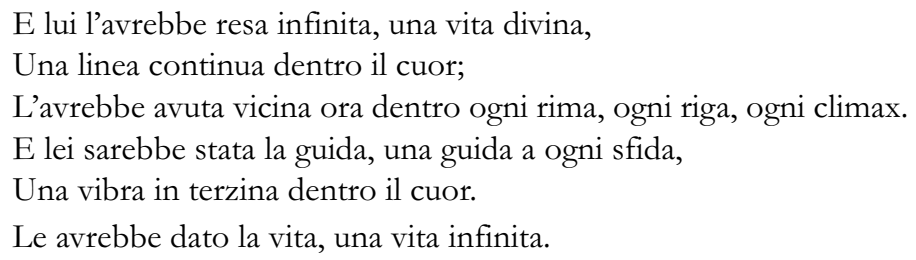

Il nome di Virgilio è solo raramente associato al ruolo di guida salvifica con il quale è ritratto nel poema dantesco: oltre al riferimento contenuto nella canzone di Murubutu e Claver Gold appena commentato (nella stessa traccia si veda anche il verso «ora che vivi in eterno hai mandato Virgilio / dal Limbo all'Inferno a ridarmi le forze», con esplicitazione dell'antroponimo), segnaliamo tra gli altri in particolare May day (2014) di En?gma, un brano attraversato da immagini fosche e popolato da figure demoniache, simbolo di passioni irrazionali che tormentano l'animo umano (cf. almeno «Assisto inerme a questo insorgere dei demoni / egemoni e crudeli contro gli angeli rinchiusi in carceri negli inferi piú macabri»), nel quale la perdita del poeta latino corrisponde, in una dimensione metaforica, allo smarrimento interiore dell'artista («Me stesso, sí, me stesso: / lotto con me stesso e spesso m'umilio / in questo viaggio dove ho perso Virgilio, / cazzo, è delirio», con inedita assonanza tra Virgilio e delirio).

Generalmente, la figura del poeta mantovano subisce invece un processo di riattualizzazione che conduce per lo piú a un suo scadimento a semplice compagno, in specie nei momenti di svago ( $«$ Saremo Dante e Virgilio che giocano a carte», Gel \& Metal Carter, I piú corrotti, 2006; «Via dal limbo su una Lambo con Virgilio», Tedua, Inferno, 2021); eccezional- 
mente si può assistere a una sua evoluzione a modello di libertinaggio, una sorta di guida licenziosa durante un incontro d'amore fortuito: «Siamo in paradiso ma è soltanto un tetto: / essersi sempre conosciuti / restando sconosciuti / solo per una notte, come due film muti: / non sono il suo amante, sono solo Virgilio» (Caneda, Angeli da bar, 2014). Tra i casi curiosi andrà infine menzionato il brano Sagrada familia, nel quale il rapper milanese Oro Bianco, in un disegno volto all'esibizione della propria agiatezza, raffigura l'insuperabile liberalità che ne caratterizza la condotta alludendo alla descrizione di avari e prodighi compiuta da Virgilio in risposta agli interrogativi di Dante in If VII (vv. 40-60): «Salto la fila il giorno del giudizio, / capo dei prodighi: dillo a Virgilio» (Oro Bianco, Sagrada familia, 2020).

\section{VOCI DALL'INFERNO}

Muovendo dall'ambito onomastico a quello piú circostanziato degli episodi del poema, l'interrogazione del corpus mostra con nitidezza l'esclusiva presenza, nei testi, di passi provenienti dall'Inferno ${ }^{55}$ In una prospettiva comparata, il dato consente di riconoscere un significativo processo di radicalizzazione a partire da un atteggiamento che era già ravvisabile negli altri generi musicali, in particolare nella canzone d'autore. ${ }^{56}$

\subsection{La selva, l'Antinferno, Caronte}

I canti piú rappresentati della cantica infernale sono il primo e il terzo. ${ }^{57}$ L'incipit del poema e l'immagine della "selva oscura" vanno considerati i riferimenti di If I maggiormente utilizzati: il verso iniziale riecheggia, almeno parzialmente, a partire dal rap della fine degli anni Novanta («Mo

\footnotetext{
${ }_{55}$ Tra le espressioni “non infernali” si segnala infatti unicamente (ma con piú d'una riserva, in quanto probabile dantismo inconsapevole) «solo soletto» («Geppetto sta a letto solo soletto in un ospizio / Mangiafuoco c'ha il tumore ma non ha piú il vizio», Caparezza, Nel paese dei balordi, 1998), forse adattata su «(anima) sola soletta» di Pg VI, 59.

${ }^{56}$ Cf. a tale proposito per lo meno Coveri 2021c: 338-44.

${ }^{57}$ Per un confronto con la canzone d'autore cf. ibid.
} 
non si vede, ma è vicino, / per noi nel mezzo del cammino», Neffa, Non tradire mai, 1998). La sua memoria si fa tuttavia piú tangibile nei prodotti del nuovo millennio; tra questi indichiamo come esempio Vendetta (2008) di Ensi: «Questa non è poesia ma vendetta in versi / nel mezzo del cammino della vita mia. / Siete persi adesso senza Ensi: / pensi di riuscire a farti strada in mezzo alla pazzia?». I brani piú interessanti tra gli ultimi in ordine di pubblicazione sono costituiti a nostro avviso da Love Kills (2014), del rapper romano CaneSecco, e Bukowski (2015), del duo Two Fingerz. Il primo si snoda a partire da una serie di riflessioni sull'amore che rende schiavi del dolore («L'amore rende schiavi del dolore tra le grida / ma non ti uccide, ti tortura e ti mantiene in vita. / L'amore è un gioco in cui ogni regola viene abolita, / e non c'è un arbitro che espelle la platea ammonita») e genera smarrimento: «Cerco un'anima gemella che mi possa rianimare, / chi sa se c'è. / Come te ho gli stessi pensieri, / sono cresciuto rimanendo un po' lo stesso di ieri: / nel bel mezzo del cammino, come Dante Alighieri, / mi sono perso tra mille domande in questi sentieri». Il secondo è un bizzarro elogio della poesia («Per portarmi a letto le ragazze / ho la tecnica Bukowski») ricalcato sul titolo italiano di un'antologia di racconti inediti di Charles Bukowsky, Scrivo poesie solo per portarmi a letto le ragazze, pubblicata dall'editore Feltrinelli nel 2012,58 che si sviluppa mediante un'elencazione di poeti e scrittori di ieri e di oggi; il nostro è ricordato tramite l'incipit della Commedia («Nel mezzo del cammino l'ho capita: male e bene vanno insieme»).

La posizione di preminenza che la "selva oscura" possiede nel complesso della concezione allegorica della Commedia dantesca - tanto per la collocazione proemiale, quanto per la fitta trama di rapporti figurali che genera nel corso del poema ${ }^{59}$ - è certamente alla base dell'enorme popolarità dell'espressione nei secoli, e del suo riuso nelle diverse forme della comunicazione, anche recenti. Per tale ragione non deve stupire che l'immagine dantesca risulti la piú fittamente disseminata nel rap: nello specifico, viene reperita in oltre trenta testi del secolo XXI, principalmente dell'ultimo decennio.

\footnotetext{
${ }^{58}$ Il titolo originale della raccolta, uscita nel 2010, è Absence of the hero.

${ }^{59}$ Cf. Sabbatini-Ragni 1970.
} 
I piú giovani esponenti del rap usufruiscono infatti della raffigurazione dello smarrimento del poeta nella fitta e misteriosa foresta per esprimere preferibilmente una condizione di crisi o, in subordine, di paura e di confusione, generate da inquietudine o disagio: «Pensavo di essere finito, invece quanto dura: / davvero ci sono finito nella selva oscura» (Giaime, Gimmi Andryx 2017 Prova 1, 2017). In alcune occasioni è associata, anche sul versante lessicale, alla nozione di "giungla", dando vita a una combinazione in parallelo che produce un effetto potenziato per la definizione di contesti metropolitani periferici e fatiscenti: «Corri, svolta nella selva oscura, / nella giungla di cemento» (Vacca, Blood a blood, 2018).

Nell'orizzonte gergale della cultura hip hop, la "selva oscura" può indicare inoltre la strada o il quartiere, la "zona", vale a dire il complesso dei punti di riferimento spaziali di uno specifico gruppo giovanile, un ambiente dotato di consuetudini condivise difficilmente codificabili al di fuori della cerchia dei membri, che appare di conseguenza sfavorevole agli "outsider" (oscura, quindi, vale qui anche 'indecifrabile'): «Il nome è Jimmy dalla selva oscura, / dove la tua legge ha meno peso di una piuma» (Rancore, Sono carzi, 2006). ${ }^{60}$

Riconosciamo infine la collocazione frequente del sintagma in rima; in tal senso non paiono privi di interesse $i$ casi nei quali viene parzialmente ripreso lo schema dantesco: «In principio venne la paura, / dopo il coraggio, poi la sete e infine l'abiura. / Nella radura, mura di tensione senza cura, / sprazzi di luce da fessura nella selva scura» (paura: scura, Murubutu, Antinferno, 2020); «E mi trovai in una selva oscura, scura, / e non sapevo piú nulla, nulla. / Perdonerai chi in amore ti trascura, scusa, / ma in fondo già lo sai, restar da solo può fare piú paura» (oscura: scura : paura, Tedua, Mare mosso, 2020). ${ }^{61}$

${ }^{60}$ Selva oscura è anche il titolo della traccia di apertura dell'album Infernum di Murubutu e Claver Gold, nella quale, attraverso un testo recitato dall'attore Vincenzo Di Bonaventura, vengono riprodotti letteralmente alcuni dei versi piú noti dei canti III, V, XIII, XXVI e XXXIV dell'Inferno.

${ }^{61}$ Cf. ancora Tedua, Due pezzi, 2020: «Il buio fa paura quando oscura la selva», con oscura forma coniugata di oscurare. Per la rima oscura: dura cf. invece il brano di Giaime, Gimmi Andryx 2017 Prova 1, supra. 
Decisamente piú rarefatta è invece la reminiscenza della "diritta via" (If I, 3): «Dico alla Digos, la CIA: "Ho solo smarrito la via". / Non hanno capito, il mio spirito è vivo, / DanTedua all'Inferno verso il Paradiso» (Tedua, Inferno, 2021). A latere si segnala ancora la ripresa, nel solo Gemon, e nella sua variante popolareggiante, di If I, 90: «I nostri muri sono spaventose distese di mattoni e di rimorsi: / benché il passato paia riproporsi, / ci siamo fatti tremare le vene ai polsi / bevendo verità a grandi sorsi» (Crimine, 2014). ${ }^{62}$

Tra i passaggi piú produttivi del terzo canto rileviamo senz'altro l'incipit, caratterizzato dalla lettura dell'iscrizione posta sulla porta dell'Inferno; la terzina d'apertura, in particolare, parrebbe costituire una soluzione efficace, nella sua trasparenza, per illustrare le atmosfere desolanti e degradate, per lo piú di collocazione urbana e periferica, che compongono la tradizionale cornice a molte delle vicende cantate dai rapper: ${ }^{63}$ "Voi siete boy scout tra le perdute genti, / fatti un tour tra le città dolenti» (DSA Commando, Spread the infection, 2013); «A me mi sale l'ansia; / tu non chiedermi: "Dov'è che si va ancora?". / Per me si va nella città dolente, Roma» (Gemitaiz, Si va pt. 2, 2015). ${ }^{64}$

Il maggior tasso di intertestualità di questa sezione ricade sul verso conclusivo dell'iscrizione (III, 9: «Lasciate ogne speranza, voi ch'intrate»), attraverso un'ampia gamma di soluzioni tematiche ed espressive, talvolta mediante vari livelli di riformulazione, indirizzate al presente: si vedano almeno i casi di Marracash («Lasciate ogni speranza voi che entrate, / l'inferno con le chiese, le righe stese, Bel Paese», Quando sarò morto, 2011), Lord Madness («Lasciate ogni speranza voi ch'entrate, che ve l'hanno già

${ }^{62}$ Sulla banalizzazione della locuzione, si vedano in particolare Frati 2010 e Serianni 2021: 70 .

${ }^{63}$ Sul rapporto tra la Commedia e alcuni scenari cittadini contemporanei si veda in particolare Pegorari 2021: 1143-63.

${ }^{64} \mathrm{Nel}$ quadro della recente canzone d'autore si vedano inoltre «Meritate di andare per me / nell'eterno dolore: / io sono il corvo Joe, / faccio paura. / State attenti, lasciatemi stare: / solo certi poeti del male / mi sanno cantare (Baustelle, Il corvo Joe, 2005) e «Sono il guardiano del Paradiso, / per me si va soltanto se sei stato buono» (Cesare Cremonini, Il pagliaccio, 2008). 
detto: / quando una storia comincia male finisce comunque peggio», $S i$ aprono le porte, 2011) e Rancore \& $\mathrm{Dj}$ Myke («Tu sei architetto sia che costruisci sia che utilizzi / la gabbia della cavia, il cartello "Lasciate ogni speranza, o voi ch'entrate nell'ignavia"», L'architetto, 2012).

L'azione pervasiva del testo dantesco sulla canzone rap contemporanea si rivela in maniera consistente anche attraverso il personaggio di Caronte. Tra gli altrii, ${ }^{65}$ il richiamo piú pregevole al traghettatore è contenuto senza dubbio nel brano a questi dedicato da Murubutu \& Claver Gold (Caronte, 2020):

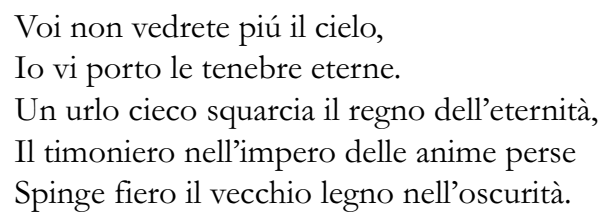

Com'è evidente, l'attacco si caratterizza per un'esplicita rielaborazione di If III, 85-87 («Non isperate mai veder lo cielo: / i’ vegno per menarvi a l'altra riva / ne le tenebre etterne, in caldo e 'n gelo»), cui segue una prima presentazione del «timoniero» (l'«urlo cieco» va con probabilità ricondotto a «gridando» di If III, 84) e della sua imbarcazione («vecchio legno» è metonimia chiaramente esemplata sul modello di «(piú) lieve legno», cf. If III, 93). Nella successiva descrizione di Caronte, cui è riservata la maggior parte della prima strofa, il testo dantesco viene sottoposto, mediante un'analoga strategia, a costante riformulazione:

Il nocchiero col remo percuote la folla: ${ }^{66}$

D'antico pelo, ${ }^{67}$ pieno d'astio e di boria

Perché tutto ciò che odia è soprattutto la sua identità.

Lo sguardo si posa anche sulla moltitudine delle anime destinate alla dannazione: «Fra i dannati mille corpi si spingevano sui bordi, / tutti in riva

${ }^{65}$ Cf. almeno Carillon (2020) di Funk Shui e Davide Shorty: «Allora, sai che ti dico guardandoti dritto con gli occhi di bracia? / Io Caronte, tu anima nera».

${ }^{66}$ Cf. If III, 110-111: «Loro accennando, tutte le raccoglie; / batte col remo qualunque s'adagia».

${ }^{67}$ Cf. If III, 83: «Un vecchio, bianco per antico pelo». 
all'Acheronte stretti a forza in mezzo ai solchi; / appena mille erano a bordo sotto i colpi di Caronte, / gli altri mille erano pronti ${ }^{68}$ verso l'Aldilà» (Caronte, vv. 5-8). Meritevole di attenzione il verso 14, «Ogni anima è foglia che cade nell'ombra», che semplifica la celebre similitudine di If III, 112114 («Come d'autunno si levan le foglie / l'una appresso de l'altra, fin che 'l ramo / vede a la terra tutte le sue spoglie»).

Derogando dal poema dantesco, i dannati acquisiscono progressivamente rilievo: nel ritornello, attraverso la presa di parola, stabiliscono infatti un contatto diretto con la voce narrante istituendo uno scambio dialogico organizzato su due turni di uguale ampiezza:

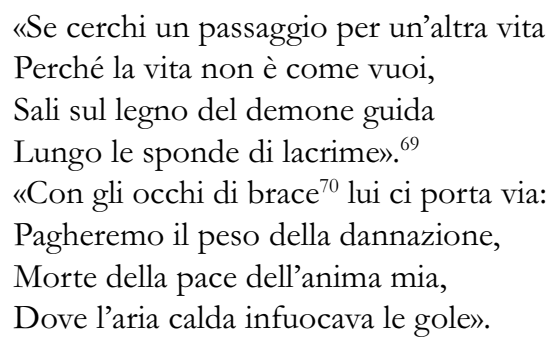

A uno dei dannati, divenuto narratore principale, si deve nella seconda strofa il mesto ritratto delle anime in attesa sulle rive dell'Acheronte:

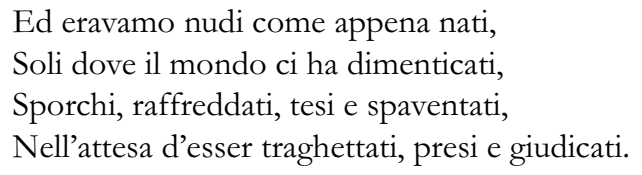

Nella sezione successiva, dalla medesima voce apprendiamo che Caronte percuote le anime con una «dura voga» (è il remo di If III, 111) ${ }^{71}$ provo-

${ }^{68}$ Cf. If III, 124: «E pronti sono a trapassar lo rio».

${ }^{69} \mathrm{Il}$ sintagma «sponde di lacrime» è forse da confrontare con «terra lagrimosa» di If III, 133.

${ }^{70}$ Cf. If III, 109.

${ }^{71}$ L'espressione riflette «due soldi per l'onore, due monete in pegno / per pagare il legno, la dura voga del traghettatore» del brano Santissima dei naufragati (2006) di Vinicio Capossela, dichiarata fonte di ispirazione per il rapper. 
cando «conati, bile, sangue e lacrime [che] si fan vapore»; la strofa acquisisce a questo punto la fisionomia di un monologo, grazie al quale il narratore ripiega su sé stesso:

Stringevo forte due monete per pagare il pegno

Per pagare il legno, ${ }^{72}$ soprattutto per sentirmi degno

Di traversare il fiume nero e poi scordare l'ero;

Sono solo un passeggero in fuga verso il nuovo regno.

La ragione della dannazione dell'anima è la dipendenza dall'eroina, l'«ero», espressamente menzionata in un unico passaggio, a fine verso e con un forte richiamo costituito dalla rima interna; il «fiume nero» è l'Acheronte (cf. «l'onda bruna» di If III, 118), ma costituisce al contempo, sul piano simbolico, un ulteriore rimando, forse meno perspicuo, al mondo della tossicodipendenza: si tratta infatti di calco parziale dell'espressione inglese black tar (lett. 'catrame nero') con la quale nel gergo della droga viene denominata una specifica varietà della sostanza stupefacente prodotta generalmente in Messico, di colore scuro ${ }^{73}$ (in Italia anche nota con il nome di eroina nera). ${ }^{74}$

${ }^{72}$ Le due monete richiamano l'obolo che come noto, secondo una tradizione popolare che proviene dalla mitologia greca e latina, ogni morto doveva pagare a Caronte affinché questi lo traghettasse nel regno dei morti. Per i riflessi dell'usanza nella tradizione letteraria italiana si rimanda a GDLI s. v. obolo' (sign. 5); cf. anche Treccani s. v. òbolo.

${ }^{73}$ Cf. https://www.addictioncenter.com/drugs/heroin/black-tar-heroin/.

${ }^{74}$ Un po' a sorpresa mancano, nel corpus analizzato, ulteriori rimandi a frammenti riconducibili al nocchiero e radicati nella memoria collettiva, quali ad es. «Non ti crucciare» (If III, 94) o «Vuolsi cosí colà» (If III, 95) o «Occhi di bragia» (If III, 109), talora produttivi, anche nel nuovo millennio, fuori dal rap, in altri generi musicali pur sempre "giovanili" o "alternativi”: cf. ad es. Afterhours, Fra i non viventi vivremo noi, 2016 («Non ti crucciare se non dormi piú, / basta una svolta e non ci sei piú»), Radici nel cemento, Bella ciccia, 2006 ("Melius abundare”: gli antichi l'hanno detto, / perciò non ti crucciare anche se ingrassi di un etto»), Modena City Ramblers, Povero diavolo, 2011 ("Caronte dimonio, / venduto il suo battello, / il suo occhio di bragia / adesso è spento») e in particolare Via Dante dei Marta sui tubi, 2005 («Vuolsi cosi colà dove si puote ciò che si vuole: / ciò che è scritto nel vento non si può piú», con successiva ripresa della prima parte del verso, quasi in funzione di "puntello": «Vuolsi cosi colà dove c'è piú di quel che pare: / c’è una gatta tra le gambe e un topolino da salvare»; «Vuolsi cosí per chi non sa cos'è / questa voglia di dire di sí che mi perseguita»). 
Ancora dal III canto, infine, sono estratte e rimaneggiate altre espressioni di matrice dantesca già saldamente radicate, talvolta in una dimensione quasi proverbiale, nella nostra lingua ad ampio raggio, tanto nel parlato quanto nello scritto, anche nelle recenti forme di comunicazione digitata; tra queste segnaliamo, per un'apprezzabile concentrazione nei testi indagati, seppur mediante riformulazioni estemporanee, soprattutto «Sanza 'nfamia e sanza lodo» (If III, 36) e «Non ragioniam di lor, ma guarda e passa» (If III, 51). ${ }^{75} \mathrm{Il}$ primo costrutto, attraverso il consueto processo di adeguamento ai canoni linguistici contemporanei, ${ }^{76}$ è recuperato, tra gli altri, da Claver Gold ne L'infezione (2021): «Paura d'esser senza lode e senza infamia, / la mia fobia è mediocrità priva di via d'uscita», con ulteriore inversione, per esigenze di rima; in un quadro affine, ma per produrre un'allitterazione in sede interna di verso, è anche in $\mathrm{Dj} 2 \mathrm{P}$, Non sei tu (2016): «Scrivo la mia trama, qui non c'è destino: / senza lode, senza infamia, quinto Celestino». Echi del verso con il quale Dante esprime, attraverso Virgilio, la propria riprovazione nei confronti degli ignavi sono invece riconoscibili, ad esempio, in Willie Peyote («Qua la gente si muove solo se le metti le mani in tasca; / qua la gente non si commuove, il mondo va a troie, guarda e passa», Incazzato lo stesso, 2011) e in Lazza ("'Zzala è un iconoclasta", dicono; "Basta, / non ti curare mai di loro, guardali e passa"», Re Mida, 2019).

Non sarà superfluo osservare che una parziale fusione dei primi due canti dell'Inferno è costituita dal già ricordato Antinferno di Murubutu e Claver Gold, un brano aperto e chiuso da un ritornello che si ripete nella composizione anche in sede interna, e che fa cosí da cornice alle due strofe, ciascuna affidata a uno dei due artisti. Il refrain è, nella sua prima parte descrittiva, una riformulazione di If III, 22-27, con inedito cenno musicale in chiusura: "Questi sospiri, pianti ed alti guai / fanno saltare l'aria senza stelle, / mentre in lacrime mi trascinai: / diverse lingue, orribili

\footnotetext{
${ }^{75}$ Per i riflessi di quest'ultima espressione negli usi recenti si rinvia in particolare all'articolo corrispondente redatto da Alessandro Aresti contenuto nell'interessante rubrica Per modo di dire. Un anno di frasi fatte curata dall'Enciclopedia Treccani consultabile al link https://www.treccani.it/magazine/lingua_italiana/articoli/parole/Modi_di_ dire10.html.

${ }^{76}$ Cf. Serianni 2021: 70-1.
} 
favelle, / musica triste senza note» ${ }^{77}$ nei tre versi finali ritorna invece il tema dell'ignavia: «Anime nude senza nome, / senza infamia e senza lode, / senza vita e senza morte». La prima strofa, interpretata da Claver Gold, colloca il viaggio nell'oltretomba, in una dimensione che si snoda, tra passato ( $« \mathrm{Ah}$, ah, in principio venne la paura, / dopo il coraggio, poi la sete e infine l'abiura») e presente («A passi lenti tra gli ulivi e bivi decisivi / tocca sfidare i morti per poi sentirci vivi, / tocca saltare i corpi, tocca schivare i corvi»), nello scenario plumbeo della selva. ${ }^{78}$ Nella seconda, Murubutu descrive l'ingresso nell'Antinferno: «Ora a passo lento, varco ed entro / sotto un cielo vasto, basso e fermo: / Antinferno, confermo, ne fermo l'immagine all'interno»; segue, in un ulteriore ritratto degli ignavi, un'ampia rielaborazione di If III $34-42^{79}$ cui si allaccia un'interrogativa sul modello di If III, 60 che conclude la sezione: «Vedi tu di chi fu il gran rifiuto e scegli: / Celestino, Esaú, Giano o Ponzio Pilato?».

\subsection{Paolo, Francesca e gli altri}

La prima eco dantesca nel rap italiano, ancora oggi una delle piú popolari, si trova come noto in Serenata rap (1994) di Jovanotti: ${ }^{80}$

$$
\begin{aligned}
& \text { Amor che a nullo amato amar perdona porco cane } \\
& \text { Lo scriverò sui muri e sulle metropolitane } \\
& \text { Di questa città, milioni di abitanti } \\
& \text { Che giorno dopo giorno ignorandosi vanno avanti. }
\end{aligned}
$$

Ciò nonostante è in definitiva contenuta, nella canzone hip hop, la memoria di Paolo e Francesca nei decenni successivi, forse poiché ritenuta,

${ }^{77}$ Cosí il testo dantesco corrispondente: «Quivi sospiri, pianti e alti guai / risonavan per l'aere sanza stelle, / per ch'io al cominciar ne lagrimai. / Diverse lingue, orribili favelle, / parole di dolore, accenti d'ira, / voci alte e fioche, e suon di man con elle».

${ }^{78} \mathrm{Cf}$. in particolare la coppia di versi «Nella radura mura di tensione senza cura, / sprazzi di luce da fessura nella selva scura» ricordata in precedenza.

${ }^{79}$ «Dentro al vestibolo vivono dietro un idolo / file d'anime in fila che da vivi non decisero: / angeli vivi? No, no; / angeli vili che alla sfida non partinne [sic] né per Dio né per Lucifero. / Sono morti senza polsi, senza opporsi, / senza porsi scopi e sogni. / Anche se vivi, già vuoti e ignari; / anche se vivi, già morti: ignavi».

${ }^{80}$ Cf. almeno Coveri 2021c: 349. 
sul piano simbolico, di minor efficacia rispetto ad altre. Quasi mai utilizzato è ad esempio il verso richiamato dall'artista di origini toscane nella sua Serenata, probabilmente il piú noto della storia dei due amanti e dell'intero canto quinto; ${ }^{81}$ ridotte al minimo sono anche le occasioni nelle quali la vicenda dei giovani viene evocata per istituire un rapporto di paragone con una specifica esperienza amorosa: «Eri pura, ed ora che sei mia sposa / sei diversa, sei piú scontrosa: / non Paolo e Francesca, ma Olindo e Rosa, / facciamo festa con vino e Prozac» (Bonnot, Storia di un imprecato, 2010, con accostamento tra le due coppie dal forte effetto dissonante); «L'odio trionfa e l'amore è un'esca, / spazzati via dal vento come Paolo e Francesca» (Lowlow, Canto V, 2017).

Alla celebre coppia è dedicato un intero brano da Murubutu e Claver Gold, Paolo e Francesca, pubblicato come singolo il 25 marzo 2020, in occasione del "Dantedî". L'ampia strofa iniziale descrive, con la voce di un narratore esterno, la «prima radice» dell'amore dei due giovani, parafrasando e ampliando il dettato dantesco di If $\mathrm{V}, 127-132$; la storia differisce da quella della Commedia per la presenza di alcuni dettagli («Là, era sera: lui leggeva e lei guardava in basso; / lei temeva quei suoi occhi») e per l'omissione dei particolari della lettura; il bacio giunge infatti all'improvviso: «Poi come in un lampo, apparso dentro allo sguardo / cauto, un bacio tremando fuggí su di lei. / E tutto lo spazio attratto dentro un abbraccio / che sfidava mille leggi e almeno cento dei». Il seguito illustra i presagi della dannazione: «Il cuore è un incendio intenso, il tempo sta fermo al centro; / rei di peccato tremendo: adulterio. / [...] / E lei sarebbe

\footnotetext{
${ }^{81}$ Piú apprezzabile è la distribuzione recente della locuzione al di fuori del perimetro del rap: oltre al noto verso di Ci vorrebbe un amico (1984) di Antonello Venditti («E se amor che a nulla ho amato / amore, amore mio perdona»), ricordiamo qui, tra le occorrenze del nuovo millennio, Un tempo indefinito (2011) di Raf («E se amor ch'a nullo amato amar perdona, / alla porta dell'inferno busserei / per poterti amare, per vederti») e soprattutto Il paese dell'amore (2018) de Lo Stato Sociale («Difendiamo fortissimo i nostri confini, / odiamo i neri ma amiamo sempre i gattini; / il nostro amore è una guerra mondiale all'italiana: / amor ch'a nullo amato, amatriciana. / Siamo io e te, tre metri in fuorigioco: / scusa ma ti chiamo errore, / che l'unica cosa seria rimasta in Italia è la ristorazione») e Sposa (2020) di Elodie, con la collaborazione di Margherita Vicario («Vanno ispirati questi uomini, / ma se li ami e glielo dici c'han paura e non perdonano, /anche quando fanno i fenomeni: / "Amor, ch'a nullo amato amar perdona”, e t'abbandonano»).
} 
andata ovunque, / basta stare insieme, / anche in un cerchio dell'Inferno, dentro un vento eterno». Il ritornello si articola in un canto in prima persona, verosimilmente attribuibile alla stessa Francesca: «Resta con me anche se non c'è un domani, / resti per me il migliore tra i peccati; /dopodiché voleremo tra i dannati, / persi dentro un cielo eterno, / al centro del nostro universo: / io muoio di te». ${ }^{82}$ Nella seconda parte del brano riconosciamo invece chiaramente la voce Paolo: «Io avevo ancora gli occhi di un bambino, / [...] / tu avevi il mare tra i capelli, sole del mattino». Il racconto del giovane parrebbe trasferire l'episodio degli amanti danteschi su un piano che trascende i limiti temporali: ${ }^{3}$ "Noi che eravamo la stessa persona, ${ }^{84} /$ principi senza corona: il tuo ricordo mi abbandona, / il tuo profumo si fa spilli, buca ed ossessiona. / Amor, ch'a nullo amato amar perdona, / e ti amo come allora»; la prospettiva infernale nella quale si colloca la loro vicenda viene tuttavia immediatamente ristabilita: «Ma qui l'inferno ci ha rubato il tempo / ci ha abbandonato dove è sempre sera: / io che mi esprimerò solo piangendo, ${ }^{85}$ / e tu parlerai di te come Ginevra».

Non va dimenticato che gli stessi Murubutu e Claver Gold hanno inoltre composto un brano che contiene, fin dal titolo, il richiamo all'altro grande personaggio del V canto, Minosse (Minosse, 2020). A differenza di quanto accaduto in altre occasioni nei componimenti dei due rapper, qui il re di Creta non agisce da protagonista: in qualità di giudice dell'Ade, viene infatti ricordato in alcuni passaggi dalla voce narrante, un dannato ${ }^{86}$

${ }^{82}$ L'immagine di Paolo e Francesca che muoiono uno dell'altra, a raffigurare un sentimento totalizzante, va forse ricondotta a If $\mathrm{V}, 106$ : «Amor condusse noi ad una morte».

${ }^{83}$ Cosí Murubutu ha in effetti presentato il brano: «Il rapporto tra Paolo e Francesca è la colpa e l'innocenza, la morale e la passione, la forma e la sostanza che attraversa i secoli, in cui tutti possiamo immedesimarci» (dal profilo Instagram murubutuofficial, al link https://www.instagram.com/p/B-KFLSfCrjo/).

${ }^{84}$ Cf. If V, 135: «Questi, che mai da me non fia diviso».

${ }^{85}$ Cf. If V, 139-140: «Mentre che l'uno spirto questo disse, / l'altro piangëa».

${ }^{86}$ Forse proprio Paolo Malatesta; si vedano infatti i seguenti passaggi del brano, nei quali il narratore si interroga sulla punizione che gli verrà inflitta: «Per me che ho amato cosí forte da perdere il fiato... / quale pena han riservato per l'amore di un dannato?»; «Io che annusavo i suoi capelli, raccoglievo i fiori, / sarò costretto per l'eterno a non sentire odori». 
che, attraversando i gironi infernali, riflette sulla natura delle pene inflitte: «La Bestia mi risputa fuori insieme ai miei dolori, / ruota la coda su se stesso e valuta i miei errori»; «Ma qua giú il vento è lussuria, Minosse s’infuria, / letame ed ingiuria, ora scendi piú giú»; «Qua tante spire quanti i cerchi scelti da Minosse»». ${ }^{87}$

Ancora dal $\mathrm{V}$ canto giungono infine al rap ulteriori schegge infernali: segnaliamo in particolare i casi di possibile reminiscenza di If $\mathrm{V}, 125$ ( caddi come corpo morto cade», dalle palesi potenzialità allitteranti): tra questi, si vedano almeno «Tende il capo color corvo, le gote bronzo sporco, / rotte le gambe cedono il colpo a un corpo morto contro un tronco / a terra sporco» (Murubutu, L'uomo senza volto, 2009) e «Fondo il mio corpo con il corpo del mic, / mi muovo anche da morto come il corpo di Mike» (Marracash, Quando sarò morto, 2011, con ulteriori richiami all'Inferno, per i quali cf. supra, che parrebbero confermare la citazione parziale del verso).

L'osservazione del corpus consente di rilevare, per gli altri rimandi infernali riscontrati nei testi, un minor tasso di circolazione. Non particolarmente pronunciate sono ad esempio le tracce dell'Ulisse dantesco: nelle canzoni rap, infatti, la reminiscenza del periplo dell'eroe greco, fittamente documentata, va quasi sempre ricondotta con evidenza all'Odissea. Di If XXVI non restano quindi che alcuni richiami, per lo piú rifatti sul v. 119 («Fatti non foste a viver come bruti»): in Argenti vive (2014), il dannato interpretato da Caparezza denuncia le contraddizioni di Dante ricordando proprio le parole da questi accordate al consigliere fraudolento: «Attaccare me non ti redime, / eri tu che davi direttive / per annichilire ogni ghibellino, / Cerchio 7, giro primo. / "Fatti non foste per vivere come bruti": / ben detta ma sputi vendetta»; in Tattattira (2015), Rancore e Dj Mike riflettono invece, in una dimensione strettamente contemporanea, sulle conseguenze determinate dall'eccessiva celebrità raggiunta da alcuni esponenti dell’hip hop, con inevitabile immiserimento del lacerto: «"Fatti non

${ }^{87}$ Dello stesso Murubutu è inoltre un ulteriore, precedente rimando al Minosse dantesco (e alla presentazione delle pene infernali); cf. infatti la La Murubeide (2009): "Qua è solo celebre cenere, remore e tenebre, / qua il re dei re dei re prese per sé in isposa Persefone, / perseguita anime perse la notte, pronte a Caronte; / anime morte, fuori i soldi da quelle bocche rotte, / poche ossa scosse chine a Minosse». 
foste a viver come bruti”: / rapper, già vendute virtute e canoscenza?».

Sul desiderio di conoscenza si concentra in maniera piú scrupolosa e profonda Ulisse (2020) di Claver Gold e Murubutu, un'efficace riscrittura della storia dell'eroe che attinge tanto all'Odissea («Ogni capello era un serpente e, mentre si fa sera, / sottocoperta conto i giorni a lume di candela. / Ora che il vento si fa grande e gonfia la mia vela, / lei sta aspettando il mio ritorno, poi disfa la tela») quanto alla Commedia ("Ora brucio con due punte in una di tre cantiche, / e gli occhi immersi tra i miei sensi che hanno sempre sete / di un folle volo ${ }^{88}$ della fantasia»). La reminiscenza specifica del poema dantesco si snoda tra i due poli della citazione puntuale («Dove ogni sbaglio era un bagaglio ricolmo di niente, / portare al limite il sapere in terre senza gente») $)^{89}$ e della libera rielaborazione («L'ottava bolgia forgia fiamme mentre il giorno muore, / dentro ogni fuoco c'è lo spirito di un peccatore; / c'è un grido stanco di dolore, la eco fa rumore, / di chi cercava nuovi mondi in acque senza amore»), in un articolato tessuto che consente al brano la conservazione di un apprezzabile livello di autonomia testuale, massima nel ritornello («Vedi, qui va tutto bene, tutto a gonfie vele, / come marinai / le onde corte. E intanto / vieni qui con gli occhi pieni: / tutto quel che vedi / a me non basta mai»).

Di sorte affine a quella di Ulisse paiono beneficiare, nel rap, Cerbero e Lucifero: si rileva infatti in diverse circostanze la tendenza all'esecuzione di generiche connessioni ai due personaggi, senza tuttavia precisi riferimenti alla lezione dantesca. Privata di corrispondenze dirette con If VI, la figura di Cerbero trova ad esempio spazio, per lo piú attraverso slittamenti metaforici, oppure in similitudini e paragoni o ancora mediante un processo di personificazione, come referente simbolico dell'aggressività umana: «Un cane a tre teste alla Cerbero, / con tutto l'odio che serbo» (Massimo Pericolo, Speranza e Barracano, Criminali, 2019); piú raramente diviene emblema di una congiuntura insidiosa: «Se c'ho un punto fisso, ora punto a perderlo: / vado dritto in bocca a Cerbero» (Gemitaiz, Dancing with the devils, 2015). Nel recente Paris (2021) di Tedua, la caratterizzazione tricefala del mostro è invece associata alle debolezze dell'artista: «In ogni

\footnotetext{
${ }^{88}$ Cf. If XXVI, 125.
}

${ }^{89}$ Cf. If XXVI, 117. 
pezzo che faccio prego un fratello condannato nel braccio, / preso da un Cerbero: droga, sesso e denaro che sperpero».

Lucifero è rappresentato in un composito ventaglio di soluzioni, talvolta anche attraverso proprietà apparentemente positive: «Io vorrei essere libero / però mi manca l'ossigeno. / Gente che pensa per me, / non ho bisogno di un re: / per loro sono Lucifero» (Nitro, Lucifero, 2018); è tuttavia indubbio che la sua collocazione agli inferi si presti per lo piú a letture figurate: «Perso tra i sogni metafisici sotto sonnifero / risplenderò nel male: vieni a svegliar Lucifero» (Claver Gold, La cicala, 2011); «Mi spingo al limite, sto su uno spigolo, / anche se mi butto, non sarò mai libero. / Mi riporta su Lucifero, / e mi riporta tra i fantasmi» (Mambolosco, Demone, 2021). Le occasioni nelle quali può essere ragionevolmente riconosciuta l'eco di If XXXIV sono limitate a Intro (2015) della Dark Polo Gang («Vestito bianco come stessi già in Paradiso / ma dietro ho Lucifero, / ali di pipistrello») $)^{90}$ e Inferno (2021) di Tedua: «Io sono un angelo caduto a terra, / non mi capacito della faccenda, / coi miei ragazzi è la pace o la guerra, / rossi i miei occhi semaforo: / Lucifero, quanti gironi che mancano?».

Ulteriori fenomeni di citazionismo dantesco vanno intesi come circostanze episodiche e rarefatte. Si ricorderà ad esempio che una delle piú discusse cruces della Commedia, il verso che contiene le parole pronunciate da Pluto nel quarto cerchio al giungere di Dante e Virgilio (If VII, 1: «Pape Satàn, pape Satàn aleppe!»), ${ }^{91}$ viene registrata esclusivamente in Machete, Non esco mai (2014): «Raramente entrate, questa è area riservata: / Pape Satan Aleppe, Pape Satan»: nell'esclamazione pare lecito riconoscere un grido di minaccia, forse l'ordine di allontanarsi da una zona insidiosa per l'interlocutore.

Entro la medesima prospettiva va riconosciuto, non senza un certo grado di sorpresa, il modesto successo del conte Ugolino (canti XXXII e

${ }^{90}$ Cf. If XXXIV, 46-51: «Sotto ciascuna uscivan due grand' ali, / quanto si convenia a tanto uccello: / vele di mar non vid' io mai cotali. / Non avean penne, ma di vispistrello / era lor modo; e quelle svolazzava, / sí che tre venti si movean da ello».

${ }^{91}$ Il riuso piú noto ed efficace del verso è di Paolo Conte: «Papé Satan, Papé Satan Aleppe, / pan e salam, pan e salam a fette» (La Java javanaise, 2000); cf. Coveri 2021c: 341. 
XXXIII), il cui ricordo risuona unicamente in Lord Madness, Like me, 2012 («Vivo in uno stato di coma continuo, / non vedo luce neanche se mi oscilli davanti agli occhi un accendino; / rispetta questo stilo, se mi vedi leva il cappellino: / cannibal rapper, Conte Ugolino») e Axos, Spectrum, 2015 («Metà bambino metà centenario, nato nel segno del vino; / bruciami in gola, mietendo, mangiando la testa: Conte Ugolino»): in entrambi è istantaneo il rimando alla feroce raffigurazione del nobile pisano mentre addenta il cranio del compagno di pena, l'arcivescovo Ruggieri (cf. in particolare XXXIII, vv. 76-78: «Quand' ebbe detto ciò, con li occhi torti / riprese 'l teschio misero co' denti, / che furo a l'osso, come d'un can, forti»). ${ }^{92}$

Una collocazione particolare spetta infine alle originali riscritture di episodi infernali evocati nel solo "concept album" Infernum di Murubutu e Claver Gold: varrà la pena ricordare in tal senso almeno i casi di Pier e Taide. Come già avveniva, almeno in parte, in Caronte, vengono utilizzati, nei brani qui ricordati, due personaggi incontrati da Dante nel corso del suo viaggio attraverso l'Inferno (If XIII e XVIII) per generare, mediante sviluppi narrativi inediti, altrettanti percorsi di riflessione sua una condizione che contraddistingue con particolare evidenza l'età contemporanea, la solitudine. Le figure dantesche svolgono quindi una funzione paradigmatica, in quanto conformi ai tormenti della sensibilità moderna: ${ }^{93}$ estratti dal contesto del poema, vengono efficacemente rivitalizzati in una nuova dimensione. $^{94}$

${ }^{92}$ Fu Fabrizio De André, come noto, il primo autore a far rivivere la vicenda di Ugolino della Gherardesca nella canzone: nel brano Carlo Martello torna dalla battaglia di Poitiers (composto insieme all'amico Paolo Villaggio), in un quadro complessivo di riuso parodico ed espressivo di forme e temi della tradizione italiana e romanza di epoca medievale, il cantautore genovese riprese infatti il celebre verso di If XXXIII, 75 («Poscia, piú che 'l dolor, poté 'l digiuno»), che conclude il racconto del conte, attraverso un calco dal forte effetto di straniamento («Ma piú che l'onor poté il digiuno») nel quale al lacerante «dolor» del padre si sostituisce, con vena maliziosa, l'«onor» del cavaliere Carlo, con conseguente risemantizzazione in chiave oscena del «digiuno» (per una lettura approfondita del brano si rimanda a Zoboli 2004: 23).

${ }^{93} \mathrm{Si}$ veda Grignani 2014: 72.

${ }^{94}$ Ibid. 
Pier, ad esempio, è un adolescente sopraffatto dall'isolamento e dalla paura («Tu che non parli ormai da tempo con gli altri bambini, / tu ti sei chiuso dentro un guscio di paure e stanco: / non hai la forza di lottare e tornare nel branco, / non hai piú voglia di sedere solo su quel banco / quando nessuno, sí, nessuno, vuole starti accanto»), vessato dai compagni di scuola («Ogni mattina chi mi invidia, chi mi insidia, / chi mi umilia, poi bisbiglia fra i maligni, cento, mille volte. / Troppe botte sulle costole, sputi ed i calci, / gli insulti degli altri, fra tutti i miei pianti. / Sempre solo contro loro, negli occhi gli sguardi; / e se mi muovo capto l'odio, le parole hanno moli giganti»). Per effetto degli atti di bullismo subiti, il giovane si toglie la vita: il suo gesto estremo, che determina l'analogia con il notaio della corte federiciana (mai esplicitamente evocato), è annunciato alla madre verosimilmente attraverso una lettera: «Quindi mamma, scusa tanto, non sono felice: / il mio cuore prende il largo da ogni sguardo ostile. / Nella stanza, sul mio banco, all'alba giú in cortile / oggi non ci sono piú, c’è un albero di vite»..$^{95}$

Mediante il recupero della memoria della cortigiana ateniese, figura minore nella Commedia, Murubutu e Claver Gold tratteggiano invece con eleganza l'animo malinconico di una giovane prostituta di oggi: «Lei si specchia tutte le sere dentro un bicchiere, / ride con le amiche mentre prende da bere; / poi si specchia di nuovo e non si riesce a piacere, / lei, che fa l'amore forse un po' per piacere, / un po' per passione, ${ }^{96}$ un po' per godere». ${ }^{97} \mathrm{Il}$ brano, che presenta un solo rimando certo al testo dantesco («Taide mente male mentre scende scale, / suona a una porta in un piano del centro: / è seconda bolgia dell'ottavo cerchio»), ${ }^{98}$ riflette in par-

${ }^{95}$ Nell'ultimo verso riprodotto non è arduo riconoscere il richiamo alla sorte dei suicidi all'interno del secondo girone del settimo cerchio dell'Inferno (cf. If XIII, 79-108).

${ }^{96}$ Probabile è qui il richiamo a Bocca di Rosa di Fabrizio De André.

${ }^{97}$ Queste le parole di Murubutu a riguardo del brano in un'intervista del 31 marzo 2000: «Volevamo utilizzare l'Inferno anche per prelevare delle figure e attualizzarle. Non volevamo essere semplicemente didascalici. Lo abbiamo fatto per prima con Taide che, per altro, è una figura decisamente minore dell'Inferno. Una figura, quella di Taide, che abbiamo recuperato e attualizzato in una prostituta» (l'intervista è leggibile al link https://archive.md/h7znI).

${ }^{98}$ Cf. If XVIII, 100-126. 
ticolare sull'inquietudine della donna, in un crescendo emozionale dominato da un'ineluttabile desolazione: "Oggi in centro Taide ha visto un uomo / che correva lungo ad una strada, / raggiungeva una ragazza sola e un suono. / Taide guarda bene mentre lei lo chiama: / intorno a loro tutto si fa sfondo, / il cuore al trotto inonda e rende i loro baci. / Lei, che per baciare chiede il doppio, / che darebbe per un bacio gratis».

\section{DANTE NELLA SELVA DEI “CULTUREMI”}

Come abbiamo cercato di documentare in queste pagine, la "potenza" dell'Inferno nei testi hip hop viene espressa attraverso un ricco catalogo di riferimenti piú o meno diretti alla cantica, che consentono, giunti a questo punto, un primo bilancio.

Nella maggior parte dei casi la citazione è "consapevole", si compone cioè di «riprese esplicite», ${ }^{99}$ prevalentemente di scolastica memoria, la cui intenzionalità è garantita da alcuni indizi certi. ${ }^{100}$ Tra questi segnaliamo naturalmente la coincidenza di un'espressione, di un sintagma, di uno o piú versi: si tratta della condizione maggiormente rappresentata; si ricorda qui, come unico esempio, la ripresa, frequente, di If III, 9 («Lasciate ogne speranza, voi ch'intrate»). Non diversamente, e con simile efficacia probatoria, agisce anche la rarità del vocabolo implicato: ${ }^{101}$ è il caso, ad es., di burella («lungo natural burelle, ${ }^{102}$ il vento divelle / ad ogni peccatore la pelle», Murubutu, Claver Gold, Lucifero, 2020). Analoghi, chiari segnali sono inoltre rappresentati dagli antroponimi, specie quelli meno popolari (cf. ad es., oltre ai precedenti, Malacoda: «Noi siamo scesi sinceri scortati da Malacoda», in Murubutu e Claver Gold, Malebranche, 2020), dai toponimi e da altri nomi geografici, a volte tra loro in combinazione (Cocito, Bruto, Cassio: «Volo sui tuoi cieli come gru nel Caspio, / nel Cocito d'astio tipo Bruto e Cassio», Claver Gold \& Kintsugi, La tana del lupo, 2019).

\footnotetext{
${ }^{99}$ Serianni 2013: 293.

${ }^{100}$ Per l'analisi delle diverse tipologie della citazione dantesca non si può prescindere da Baranski 1986: 343-76.

${ }^{101}$ Cf. Serianni 2013: 293.

${ }^{102}$ Cf. If XXXIV, 98.
} 
La "consapevolezza" viene inoltre garantita dalla presenza, nel medesimo contesto entro il quale si colloca una citazione, di altri richiami danteschi concomitanti; in questo modo va interpretata in particolare la convergenza di parole in rima, nel corpus ben illustrata, tra le altre, dalle sequenze (selva) oscura: paura (Murubutu, Antinferno, 2020 e Tedua, Mare mosso, 2021) e (selva) oscura : dura (Giaime, Gimmi Andryx 2017 Prova 1, 2017). Occorre evidentemente inserire in questo raggruppamento anche i numerosi e multiformi episodi di riformulazione (di una voce, di un sintagma, di un verso): «Questi sospiri, pianti ed alti guai / fanno saltare l'aria senza stelle / mentre in lacrime mi trascinai / diverse lingue, orribili favelle, / musica triste senza note» (Murubutu e Claver Gold, Antinferno, 2020).

Accanto alle riprese esplicite, ma pur sempre entro il confine della citazione intenzionale, riconosciamo inoltre i casi, a ben vedere non cosí frequenti, di richiamo a un episodio infernale in assenza di tracce di versi o di altri elementi linguistici di palese matrice dantesca (si ricordi a tale proposito il brano Pier); nel perimento di questa tipologia, che potremmo definire "indiretta", individuiamo ancora le numerose allusioni, specie per finalità espressive (cf. almeno il Caparezza di Azzera pace riprodotto in apertura di contributo).

A un diverso livello di consapevolezza si colloca infine la tecnica della riscrittura, messa efficacemente a frutto nell'antologia infernale di Murubutu e Claver Gold, un progetto senza dubbio complesso e raffinato che esplora e combina i percorsi offerti dalle diverse soluzioni fin qui presentate definendo una mappa tematica e stilistica inedita nel panorama del rap e della canzone italiana recente.

Non sono d'altro canto infrequenti i dantismi che potremmo definire, ancora con Serianni, "inavvertiti”, ${ }^{103}$ vale a dire quell'ampia gamma di lessico (singole parole, sintagmi, espressioni) e fraseologia caratterizzata da origine dantesca «obliterata» ${ }^{104}$ per effetto della popolarità acquisita, capace quindi di spaziare liberamente nei diversi ambiti d'uso della lingua. Tra i numerosi esempi di citazione inconsapevole, di minore interesse per l'indagine in corso, ci limitiamo a segnalare qui almeno la presenza dei tipi

\footnotetext{
103 Serianni 2021: 73 .

${ }^{104}$ Ibid.
} 
«non mi tange» (Willie Peyote, Interludio (L'Avvelenata), 2015: «L'ipocrisia non mi tange, / è una guerra fra poveri, io non prendo il cachet, prendo le mance») e «stai fresco» (Rancore, Rapper al rovescio, 2016: «A bassa voce gli ho risposto: "Se, cosí stai fresco", / presto, la rabbia si delinea, se oggi finalmente esco»), entrambi attestati anche in altre forme coniugate, le «dolenti note» (sempre in rapporto alla musica: Mondo Marcio, Il Paradiso perduto, 2004: «A volte mi chiedo perché suono queste dolenti note»; con inversione in Truceboys, Il giardino degli Dei, 2003: «Ora vivo ossessionato dallo schermo, / soffoco la notte, note dolenti») e il «bel paese» (Fabri Fibra, Su le mani 2016, 2016: «Qui son tutti matti, questo è il Belpaese»). ${ }^{105}$

Non andrà trascurato, in chiusura, un ultimo aspetto reputato significativo, rimasto - nel corso di queste riflessioni - in penombra, o affiorato solo in maniera episodica: lo scarto, che definiremmo ampio, tra il rap e gli altri generi musicali, compresa la corrente cantautorale, nell'attitudine al dialogo con la Commedia: se è infatti innegabile che anche attraverso un prodotto della cultura di massa come la canzone «nessun poeta come Dante, facendo parlare i morti, ha parlato e continua a parlare ai vivi», ${ }^{106}$ è allo stesso modo incontestabile che in nessun altro genere musicale al di fuori del nostro la voce del poeta è risuonata e continua a risuonare, insieme a quelle delle anime dell'Inferno, con simile vitalità.

Il dato non deve stupire: il massimo grado di intertestualità dantesca rilevabile nell'hip hop, e in particolare nella produzione degli ultimi anni, va posto in diretta dipendenza di alcuni dei piú caratteristici tratti costitutivi del genere richiamati in apertura.

Anzitutto, se l'immaginario dantesco è divenuto in larga parte universale ed emblematico, nei personaggi e negli episodi infernali alcuni rapper hanno potuto riconoscere, piú che in ogni altra possibile contingenza, figure e ambienti simbolici, comportamenti e situazioni "possibili", in grado di stabilire connessioni con il mondo contemporaneo - meglio, con gli

105 Ma cf. anche Caparezza, Goodbye malinconoia (2011): «E pensare che per Dante questo era il "bel paese là dove 'l sí sona"» e Inoki, Non mi avrete mai, 2005: «Fratello, svegliati e guarda questa Italia, / il Belpaese dove non hai opportunità».

${ }^{106}$ L'espressione, non applicata alla canzone, è tratta da Beccaria 2021: 148. 
inferni contemporanei descritti in molti brani -, quindi di ricostruire all'occorrenza, anche nella stretta attualità della strada e della periferia, un'ampia gamma di sfumature di sentimenti, passioni e temperamenti, dall'amore all'odio, dall'audacia alla paura, dalla speranza alla disperazione.

Sul fronte stilistico, poi, ha certamente agito in tal senso il ricorso costante a una configurazione metrica fissa, o per lo meno caratterizzata da un assetto regolare, le cui esigenze determinano la preferenza per soluzioni ritmiche che sono proprie della poesia.

Si ritiene infine che un ruolo determinante sia stato svolto dalla costante ricerca della sperimentazione linguistica connaturata al genere, ottenuta, tra l'altro, dalla commistione degli stili e dall'incessante riuso di materiali di diversa provenienza, con conseguente "cortocircuitazione" di modelli alti e bassi. Nei testi dei rapper di ultima generazione si percepisce in particolare - con una concentrazione maggiore rispetto a quella del rap "di strada" delle origini - una sorta di esibizione di conoscenza che può sfociare a volte in una "sindrome" da citazionismo spasmodico: il testo appare cosí reiteratamente costellato di elementi eterogenei, spesso provenienti da codici culturali disparati, una selva fitta di "culturemi", ${ }^{107}$ che definiscono uno scenario intricato e talvolta farraginoso nel quale il nostro, a ben vedere, può correre ancora una volta il rischio di smarrirsi.

Luca Bellone

(Università degli Studi di Torino)

\section{RIFERIMENTI BIBLIOGRAFICI}

Accademia degli Scrausi 1996 = Accademia degli Scrausi (a c. di), Versi rock. La lingua della canzone italiana negli anni '80 e '90, Milano, Rizzoli, 1996.

Antonelli 2005 = Giuseppe Antonelli, Il complesso pop. Su una tendenza recente dei testi di canzone, in Elisa Tonani (a c. di) Storia della lingua italiana e storia della musica, Firenze, Cesati, 2005: 219-32.

\footnotetext{
${ }^{107}$ Sul concetto di “culturema” si rimanda soprattutto a Luque Nadal 2009: 93-120.
} 
Antonelli $2010=$ Giuseppe Antonelli, Ma cosa vuoi che sia una canzone. Mezzo secolo di italiano cantato, Bologna, il Mulino, 2010.

Antonelli-Milone 2021 = Giuseppe Antonelli, Federico Milone, Dante pop, in Frosini-Polimeni 2021a: 318-28.

Baranski 1986 = Zygmunt G. Baranski, The Power of Influence: Aspects of Dante's Presence in Twentieth-century Italian Culture, «Strumenti Critici» n. s. 1 (1986): 343-76.

Beccaria 2021 = Gian Luigi Beccaria, Dante vicino, settecento anni dopo, in FrosiniPolimeni 2021a: 148-58.

Bellone 2017 = Luca Bellone, «Tu a me non mi canti. Mi faccio cantare dal De André»: decostruzione del canone letterario e altre "provocazioni" ne «Le Beatrici» di Stefano Benni, in Aa.Vv., Quaderni di studi italiani e romeni, vol. VII, Alessandria, Edizioni dell'Orso, 2016 (2017): 101-21.

Bravi 2020 = Francesca Bravi, La «Marina Commedia» di Vinicio Capossela. Canzone d'autore e letteratura di viaggi in "Marinai, profeti e balene», in Coveri-Diadori 2020: 59-72.

Cartago 2008 = Gabriella Cartago, La lingua della canzone, in Ilaria Bonomi, Andrea Masini, Silvia Morgana (a c. di), La lingua italiana e i mass media (2003), Roma, Carocci, 20087: 199-221.

Casadei 2013 = Alberto Casadei, Dante nel ventesimo secolo (e oggi), in Id., Dante oltre la Commedia, Bologna, il Mulino, 2013: 145-80.

Casadei 2020 = Alberto Casadei, Dante. Storia avventurosa della «Divina Commedia» dalla selva oscura alla realtà aumentata, Milano, il Saggiatore, 2020.

Casadio 1996 = Gianfranco Casadio, Dante nel cinema, Ravenna, Longo, 1996

Ciabattoni 2016 = Francesco Ciabattoni, La citażione è sintomo d'amore. Cantautori italiani e memoria letteraria, Roma, Carocci, 2016.

Commedia $($ Chiavacci Leonardi) $=$ Dante Alighieri, Commedia, con il commento di Anna Maria Chiavacci Leonardi, Milano, Mondadori, 1991 (If), 1994 (Pg), 1997 (Pd), 3 voll.

Conti 2021 = Fulvio Conti, Il sommo italiano. Dante e l'identità della nazione, Roma, Carocci, 2021.

Cotugno 2009 = Anna Maria Cotugno, Dante a fumetti, Foggia, Edizioni del Rosone, 2009 .

Cotugno-Gargano 2016 = Anna Maria Cotugno, Trifone Gargano, Dante pop: romanzi, parodie, brand, canzoni, Bari, Progedit, 2016.

Coveri 1992 = Lorenzo Coveri, Dallo scritto al cantato: litaliano della canzonetta, in Aa.Vv., Gli italiani scritti, Incontri del Centro di studi di grammatica italiana. Firenze, 22-23 maggio 1987, Firenze, Accademia della Crusca, 1992: 15382. 
Coveri 1996 = Lorenzo Coveri, Per una storia linguistica della canzone italiana, in Id., Parole in musica. Lingua e poesia nella canzone d'autore italiana, Novara, Interlinea, 1996.

Coveri 2011 = Lorenzo Coveri, Le canzoni che hanno fatto l'italiano, in Elisabetta Benucci, Raffaella Setti (a c. di), Italia linguistica: gli ultimi 150 anni. Nuovi soggetti, nuove voci, un nuovo immaginario, Firenze, Accademia della Crusca $\cdot$ Le Lettere, 2011: 69-126.

Coveri 2021a = Lorenzo Coveri, Dante 2021. Cantare Dante, da Petrolini a De André, da Jovanotti a Fedez, tra rock e poesia, «Mentelocale Magazine», 25 marzo 2021, consultabile al link https://www.mentelocale.it/magazine/articoli/88388dantedi-2021-cantare-dante-petrolini-de-andre-jovanotti-fedez-rock-poesia.htm.

Coveri 2021b = Lorenzo Coveri, Dante 700: di tutto un pop, «Andersen» (Mensile di letteratura e illustrazione per il mondo dell'infanzia) 382 (2021): 12-5.

Coveri 2021c = Lorenzo Coveri, Dante nelle canzoni, in Frosini-Polimeni 2021a: $336-69$.

Coveri-Diadori 2020 = Lorenzo Coveri, Pierangela Diadori (a c. di), Litaliano lungo le vie della musica: la canzone, Firenze, Cesati, 2020.

Cristalli 2019 = Beatrice Cristalli, Milano non è una città, è una_Cosa, quarta puntata dello speciale Canzoni e parole nei cuori dellitpop, Treccani, consultabile al link https://www.treccani.it/magazine/lingua_italiana/articoli/percorsi/percorsi_195.html.

De Martino 2013 = Delio De Martino, Dante e la pubblicità, Bari, Levante, 2013.

De Rooy 2003 = Ronald De Rooy, «Il poeta che parla ai poeti». Elementi danteschi nella poesia italiana ed anglosassone del secondo Novecento, Firenze, Cesati, 2003.

Della Corte 2021 = Federico Della Corte, Canzoni dall'Inferno, speciale della sezione La lingua italiana, Treccani, consultabile al link https://www.treccani. it/magazine/lingua_italiana/speciali/Dantesi/03_Della_Corte.html.

Frati 2010 = Angela Frati, Da far tremar le vene e i polsi!, scheda di Consulenza linguistica, Accademia della Crusca, 2010, consultabile al link https://accademiadellacrusca.it/it/consulenza/da-far-tremar-le-vene-e-i-polsi/276.

Frosini-Polimeni 2021a = Giovanna Frosini, Giuseppe Polimeni (a c. di), Dante, l'italiano, Accademia della Crusca, Firenze, 2021 (edizione digitale Firenze, goWare).

Frosini-Polimeni 2021b = Giovanna Frosini, Giuseppe Polimeni, Dante per il mondo, in Frosini-Polimeni 2021a: 6-8.

Galvagni 2012 = Fabrizio Galvagni, Dante e l'armonia delle sfere. La «Commedia», il rock progressivo e altri percorsi, Milano, Vololibero, 2012.

Gargano 2018 = Trifone Gargano, Dante pop. Canzoni e cantautori, Bari, Progedit, 2018. 
Gargano 2021 = Trifone Gargano, Dante pop e rock. Le suggestioni dantesche nella musica e nella cultura, Bari, Progedit, 2021.

$G D L I=$ Grande Dizionario della Lingua Italiana, diretto da Salvatore Battaglia, Torino, UTET, 1961-2001, 21 voll.

Grignani 2014 = Maria Antonietta Grignani, Presenze della «Divina Commedia» nella poesia del Novecento, in Margherita Quaglino, Raffaella Scarpa (a c. di), Metodi. Testo. Realtà. Atti del Convegno di Studi, Torino, 7-8 maggio 2013, Alessandria, Dell'Orso, 2014: 69-100.

Guerra 2020 = Davide Guerra, «Paolo e Francesca quelli io me li ricordo bene». Echi danteschi nella canzone italiana, Pasturana (AL), Puntoacapo editrice, 2020.

Luque Nadal 2009 = Lucía Luque Nadal, Los culturemas: ¿ unidades lingüisticas, ideológicas o culturales?, «Language Design» 11 (2009): 93-120.

Madrussan 2021 = Elena Madrussan, Formazione e musica. L'ineffabile significante nel quotidiano giovanile, Milano · Udine Mimesis edizioni, 2021.

Miglietta 2019 = Annarita Miglietta, Sulla lingua del rap italiano. Analisi quali-quantitativa dei testi di Caparezza, Firenze, Cesati, 2019.

Pegorari $2000=$ Daniele Maria Pegorari, Vocabolario dantesco della lirica italiana del Novecento, Bari, Palomar, 2000.

Pegorari $2010=$ Daniele Maria Pegorari, $«$ Per dire» la storia: Dante nella prosa contemporanea, «Dante. Rivista internazionale di studi su Dante Alighieri» 7 (2010): 115-47.

Pegorari 2012 = Daniele Maria Pegorari, Il codice Dante. Cruces della «Commedia» e intertestualità novecentesche, Bari, Stilo, 2012.

Pegorari 2018 = Daniele Maria Pegorari, «La pioggia continua converte in bozzacchioni le sosine vere»: sui rischi del dantismo 'di massa', «Dante. Rivista di studi su Dante Alighieri» 15 (2018): 77-81.

Pegorari 2021 = Daniele Maria Pegorari, Inferni metropolitani: tracce dantesche nella rappresentazione della città (1955-1975), «Critica letteraria» 192-193 (2021): 1143-63.

Pertile $2021=$ Lino Pertile, Dante popolare, Ravenna, Longo, 2021.

Polese 2019 = Ranieri Polese, Tu cbiamale se vuoi... Citazioni, echi, lasciti letterari nelle canzoni italiane, Milano, Archinto, 2019.

Sabbatini-Ragni 1970 = Mirella Sabbatini, Eugenio Ragni, Selva, in Aa.Vv., Enciclopedia Dantesca, diretta da Umberto Bosco, Roma, Istituto della Enciclopedia Italiana Treccani, 1970-1978, 6 voll. (la voce è leggibile al link https:/ / www.treccani.it/enciclopedia/selva_\%28Enciclopedia-Dantesca\%29/).

Scholz $2006=$ Arno Scholz, Subcultura e lingua giovanile in Italia. Hip-hop e dintorni, Roma, Aracne, 2006.

Scorrano 1976 = Luigi Scorrano, Modi ed esempi di dantismo novecentesco, Lecce, Adriatica editrice salentina, 1976. 
Scorrano 1994 = Luigi Scorrano, Presenza verbale di Dante nella letteratura italiana del Novecento, Ravenna, Longo, 1994.

Serianni 2013 = Luca Serianni, Echi danteschi nell'italiano letterario e non letterario, «Italica» 90/2 (2013): 290-8.

Serianni 2021 = Luca Serianni, Parola di Dante, Bologna, il Mulino, 2021.

Talanca 2017 = Paolo Talanca, Il canone dei cantautori italiani. La letteratura della canzone d'autore e le scuole delle erà, Lanciano, Carabba, 2017.

Treccani $=$ Aa.Vv., Vocabolario on line, Istituto della Enciclopedia Italiana Treccani, consultabile al link https://www.treccani.it/enciclopedia/elenco-opere/Vocabolario_on_line.

Vita Nuova (Pirovano-Grimaldi) = Dante Alighieri, Vita Nuova. Rime, edizione a cura di Donato Pirovano, Marco Grimaldi, introduzione di Enrico Malato, Tomo I, Vita Nuova. Le rime della «Vita Nuova» e altre rime del tempo della «Vita Nuova», Roma, Salerno Editrice, 2015.

Zoboli 2004 = Paolo Zoboli, De André, Carlo Martello e la "pastorella”, «Trasparenze» 22 (2004): 51-69.

Zuliani 2018 = Luca Zuliani, L'italiano della canzone, Roma, Carocci, 2018.

RIASSUNTO: L'indagine intende fornire - giunti ormai quasi al termine delle celebrazioni per i settecento anni dalla morte del poeta - un nuovo contributo allo studio della ricezione dantesca nella canzone italiana, focalizzando l'attenzione sui principali meccanismi che regolano la citazione della Commedia nel rap.

Parole Chiave: Dante Alighieri, Divina Commedia, intertestualità dantesca, italiano della canzone, filologia italiana.

ABSTRACT: The essay intends to provide a new contribution to the study of Dante's reception in Italian song, focusing on the main mechanisms that govern the quotation of the Comedy in rap.

Keywords: Dante Alighieri, Divina Commedia, Dante's intertextuality, Italian language in songs, Italian philology. 